\title{
Dectin-3 Recognizes
}

Glucuronoxylomannan of

Cryptococcus neoformans

Serotype AD and Cryptococcus

gattii Serotype B to Initiate Host Defense Against Cryptococcosis

OPEN ACCESS

Edited by:

Celio Geraldo Freire-de-Lima,

Universidade Federal do Rio de

Janeiro, Brazi

Reviewed by:

Charley Christian Staats,

Universidade Federal

do Rio Grande do Sul

(UFRGS), Brazil

Juliana Dutra Barbosa Da Rocha,

University of Toronto, Canada

*Correspondence:

Xin-Ming Jia

jiaxm@tongji.edu.cn

Jin-Fu Xu

jfxucn@gmail.com

tThese authors have contributed equally to this work.

Specialty section: This article was submitted to

Microbial Immunology,

a section of the journal

Frontiers in Immunology

Received: 27 April 2018

Accepted: 19 July 2018

Published: 06 August 2018

Citation:

Huang H-R, Li F, Han H, XuX, Li N,

Wang S, Xu J-F and Jia X-M

(2018) Dectin-3 Recognizes

Glucuronoxylomannan of

Cryptococcus neoformans

Serotype $A D$ and Cryptococcus gattii Serotype $B$ to Initiate Host Defense Against Cryptococcosis.

Front. Immunol. 9:1781. doi: 10.3389/fimmu.2018.01781

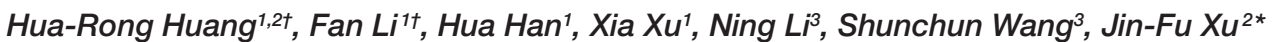 \\ and Xin-Ming Jia ${ }^{1 *}$
}

\begin{abstract}
Shanghai Skin Disease Hospital, Tongii University School of Medicine, Shanghai, China, ${ }^{2}$ Department of Respiratory and Critical Care Medicine, Shanghai Pulmonary Hospital, Tongji University School of Medicine, Shanghai, China, ${ }^{3}$ Institute of Chinese Materia Medica, Shanghai University of Traditional Chinese Medicine, Shanghai, China
\end{abstract}

Cryptococcus neoformans and Cryptococcus gattii cause life-threatening meningoencephalitis or lung diseases in immunocompetent individuals or immunocompromised ones. C. neoformans and C. gattii are subdivided into five serotypes based on their capsular glucuronoxylomannan (GXM). C. neoformans consists of serotypes $A$, $D$, and $A D$ hybrid, and C. gattii consists of serotypes B and C. Given structural differences of GXM between $C$. neoformans and $C$. gattii, it remains unclear that how innate immune system recognizes GXM. Here, we report that C-type lectin receptor Dectin-3 (MCL encoded by $\mathrm{Clec} 4 \mathrm{~d}$ ) is a direct receptor for GXMs from $C$. neoformans serotype $A D(C . n-A D)$ and C. gattii serotype B (C.g-B). GXMs from C.n-AD and C.g-B activated NF-kB and ERK pathways to induce pro-inflammatory cytokine production, whereas it was completely abolished due to deficiency of Dectin-3 or caspase recruitment domain family member 9 (CARD9). Upon pulmonary C.n-AD and C.g-B infection, Dectin-3- and CARD9-deficient mice were highly susceptible and showed augmented lung injury due to impairment of alveolar macrophage accumulation and killing activities. Our study provides the first biological and genetic evidence demonstrating that Dectin-3 recognizes GXM of C.n-AD and C.g-B to initiate host defense against cryptococcosis.

Keywords: innate immunity, C-type lectin receptor, Dectin-3, Crytococcus, glucuronoxylomannan

\section{INTRODUCTION}

The saprophytic, encapsulated fungal pathogens Cryptococcus neoformans and Cryptococcus gattii can cause life-threatening meningoencephalitis and pneumonia in both immunocompromised and immunocompetent individuals, which is called cryptococcosis (1). C. neoformans has been classified into three serotypes including $\mathrm{A}, \mathrm{D}$, and $\mathrm{AD}$ hybrid whereas serotypes $\mathrm{B}$ and $\mathrm{C}$ have been recognized as a separate species called $C$. gattii based on antigenic differences in the polysaccharide capsules of the fungus (2-4). C. neoformans serotype A (C.n-A) is the most clinically prevalent in immunocompromised individuals, including HIV patients, renal transplant recipients, and those undergoing immunosuppressive therapy (5), whereas C. neoformans serotype D 
(C.n-D) is mostly found in Europe but has a sporadic global distribution (6). A recent survey in Europe revealed that 19\% of human infections are caused by $C$. neoformans serotype $\mathrm{AD}$ (C.n-AD), which is a hybrid of serotype A and D strains (7). In contrast, infection by $C$. gattii including serotype $B$ and C (C.g-B and C.g-C) is much less common in immunocompromised patients but is thought to be more virulent than C. neoformans and causes disseminated infections even in healthy hosts (8). Previously, it was thought that C. gattii infections were restricted to tropical and subtropical regions (9), but the emergence of the outbreak events due to C. gattii infections in temperate areas of North America suggest a more global distribution of this yeast $(10,11)$.

Both C. neoformans and C. gattii are found ubiquitously in soil and other niches, and inhalation of yeast or desiccated basidiospores into the lungs is extremely common, causing about $70 \%$ of children with pulmonary infections in urban environments (12). Host immune cells including alveolar macrophages (AMs) and dendritic cells (DCs) recognize pathogenassociated molecular patterns via pattern-recognition receptors (PRRs), which elicit the host defense response. C-type lectin receptors (CLRs), a PRR-recognizing PAMP composed of polysaccharides, have garnered the attention of many investigators in the study of host defense against fungal infection $(13,14)$. The genetic deficiency of Dectin-1, a representative CLR recognizing $\beta 1$,3-glucans of Candida albicans yeast and Aspergillus fumigatus conidia $(15,16)$, did not influence the clearance of C.n-A pulmonary infections (17). Dectin-2 is known to recognize $\alpha$-1,2-mannans of $C$. albicans and A. fumigatus hyphae, lipophilic and hydrophilic components of Malassezia, mannosecapped lipoarabinomannan of Mycobacterium tuberculosis and unknown components of non-capsular C. $n$-A to trigger the production of various cytokines and chemokines, including pro-inflammatory Th1, Th17, and also Th2 cytokines (18-22). Our earlier studies show that Dectin-3 (also called CLECSF8, MCL, or Clec4d) can recognize $\alpha-1,2$-mannans of C. albicans hyphae and trehalose 6,6'-dimycolate of $M$. tuberculosis $(23,24)$. However, a recent study shows that Dectin-3 is dispensable for mediating protective immune responses against pulmonary C.n-A infection (25). The genetic defect of the caspase recruitment domain family member 9 (CARD9), an adaptor protein that operates downstream of CLRs for activating NF- $\mathrm{KB}$ and extracellular signal-regulated protein kinase (ERK) pathways $(26,27)$, confers susceptibilities to pulmonary C.n-A infection probably due to the reduced accumulation of IFN- $\boldsymbol{\gamma}$-expressing NK and memory T cells (28).

Cryptococcal capsule is composed primarily of glucuronoxylomannan (GXM), which comprises more than $90 \%$ of the capsule's polysaccharide mass (29). The typical GXM consists of a linear $(1 \rightarrow 3)$ - $\alpha$-D-mannopyranan bearing $\beta$-D-xylopyranosyl (Xylp), $\beta$-D-glucopyranosyluronic acid, and 6-O-acetyl substituents (30-32). The disposition of the $\mathrm{O}$-acetyl substituents is the major determinant of the antigenic activity observed among GXMs obtained from all serotypes (A, B, C, D, and AD) (33). The ability of GXM to activate the toll-like receptor (TLR)-mediated innate immune response has been reported in several studies (34-36). In detail, GXM from C.n-A activates
TLR4-mediated intracellular signaling (34), but its contribution to the global innate response against $C$. neoformans infections is limited $(37,38)$. GXM from C.n-A can also interact with TLR2 (34), which is believed to influence the response to cryptococcal infection (35). A recent study shows that GXM from five cryptococcal serotypes were differentially recognized by TLR2/TLR1 and TLR2/TLR6 heterodimers (36). Overall, most studies on the immunological functions of GXM have focused on the polysaccharide fractions from C.n-A isolates. Given the structural differences of GXM among the five serotypes, it also remains unknown that how host innate immune system differentially recognizes these GXMs.

In the present study, we show that Dectin-3 is a direct receptor for capsular GXM from C.g-B and C.n-AD, but not C.n-A, C.n-D, and C.g-C. Furthermore, we demonstrate that Dectin-3 is essential for GXM-induced inflammatory responses through activating NF- $\mathrm{BB}$ and ERK pathways. Therefore, Dectin-3-deficient mice are highly sensitive to pulmonary C. $g$-B and C.n-AD infections due to impairment of alveolar macrophage activation.

\section{RESULTS}

\section{Dectin-3 Recognizes the Capsule of C.g-B to Induce NF-KB and ERK-Mediated Pro-Inflammation Responses}

In nature and under laboratory conditions, the capsule of Cryptococcus strains is relatively thin but can be mature to become thick during mammalian infection (39). In this study, C.g-B strain ATCC32609 was prepared as thin- and thick-capsulated suspensions as described (39) and capsule induction was confirmed by India ink staining (Figure 1A). To explore whether Dectin-3 is required for C.g-B induced pro-inflammation responses, we stimulated bone marrow-derived macrophages (BMDMs) from wild-type (WT, Clec4d $\left.{ }^{+/+}\right)$and Dectin-3-deficient $\left(\mathrm{Clec}^{-/-}\right)$ mice with thin- and thick-capsulated C.g-B, and found that both thin- and thick-capsulated C.g-B strongly induced nuclear translocation of NF- $\kappa \mathrm{B}$ (p65 subunit) (Figure 1B), together with $\mathrm{I} \kappa \mathrm{B} \alpha$ phosphorylation and degradation (Figure 1C). In contrast, Dectin-3 deficiency completely impaired the C.g-B-induced $\mathrm{NF}-\kappa \mathrm{B}$ nuclear translocation (Figure 1B) and IкB $\alpha$ phosphorylation and degradation (Figure 1C). Moreover, both thin- and thickcapsulated C.g-B triggered sustained phosphorylation of ERK in WT BMDMs (Figure 1C), but Dectin-3 deficiency dramatically impaired C.g-B-induced ERK activation (Figure 1C). Together, these results indicate that Dectin-3 is critical for C.g-B-induced activation of NF- $\kappa \mathrm{B}$ and ERK pathways. Furthermore, we found that secretion of pro-inflammation cytokines including TNF- $\alpha$ and IL-6 were increased in WT BMDMs when stimulated with thin- and thick-capsulated C.g-B, whereas Dectin-3 deficiency significantly impaired these responses (Figure 1D). However, C.g-B-induced levels of IL-12p40 and IL- $1 \beta$ were comparable in WT and Dectin-3-deficient BMDMs (Figure 1D). These results suggest that Dectin-3 is essential for C.g-B-induced NF- $\kappa$ B and ERK-mediated pro-inflammation responses.

During cryptococcal infection, various chemokines are produced by alveolar monocytes to attract inflammatory cells 
A
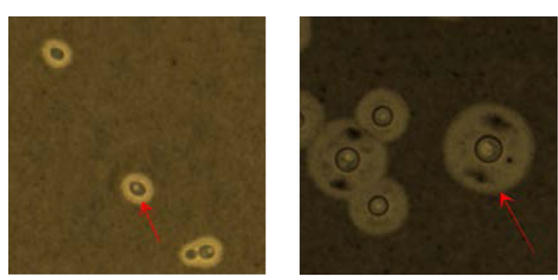

B

C. $g-B$

(h)

$0 \frac{\text { Thin-cap }}{12} \frac{\text { Thick-cap }}{1 \quad 2} 0 \frac{\text { Thin-cap }}{1 \quad 2} \frac{\text { Thick-cap }}{1 \quad 2}$

p65

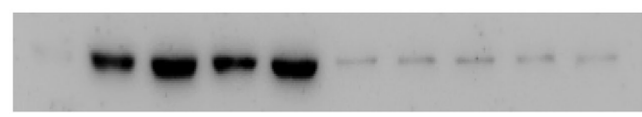

PCNA

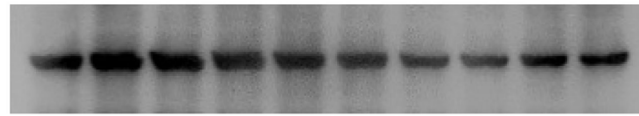

Clec $4 \mathrm{~d}^{+/+}$
C

C. $g$ - $B$
(min) $\quad 0 \quad \frac{\text { Thin-cap }}{153045} \frac{\text { Thick-cap }}{1530450} 0 \frac{\text { Thin-cap }}{153045} \frac{\text { Thick-cap }}{153045}$

p-IkBa

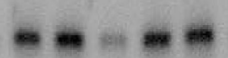

IkBa

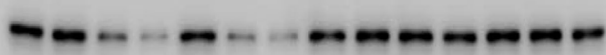

p-ERK

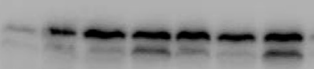

ERK1

Actin

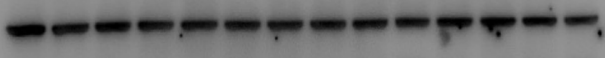

Clec $4 \mathrm{~d}^{+/+}$

D
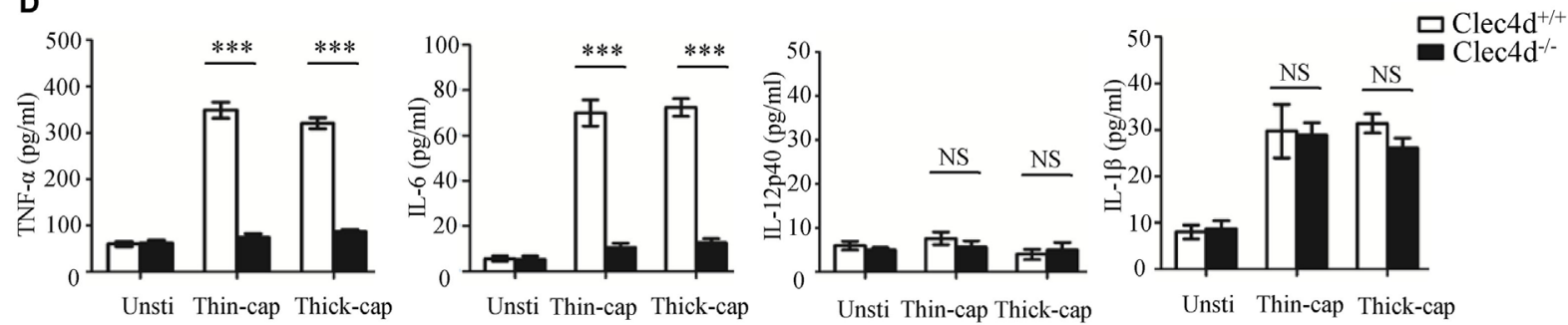

E
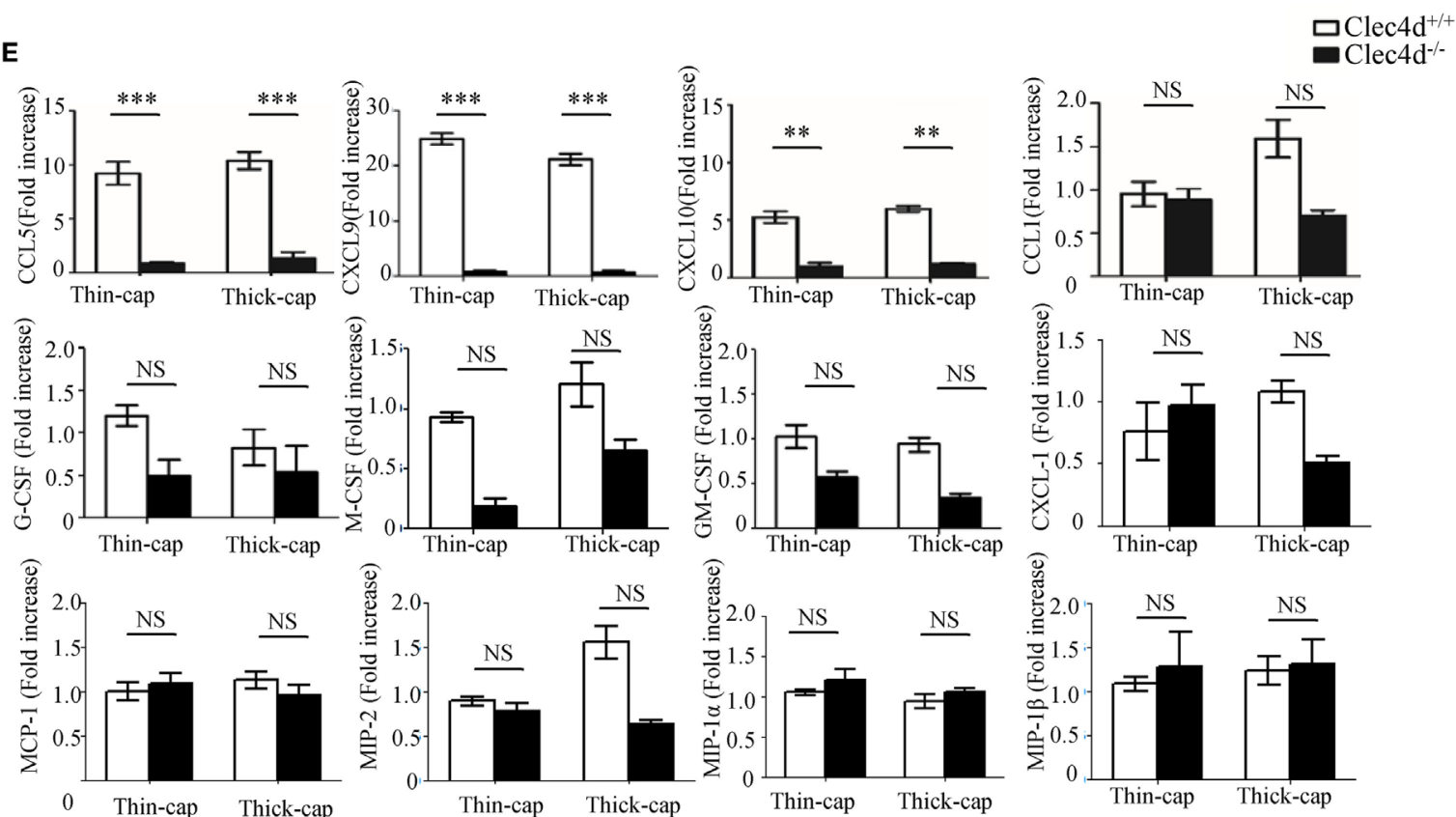

FIGURE 1 | Dectin-3 is essential for Cryptococcus gattii-B-induced NF-kB and ERK-mediated pro-inflammation responses. (A) C.g-B strain ATCC32609 was cultured with thin-capsule form in yeast extract peptone dextrose medium at $30^{\circ} \mathrm{C}$ and induced into thick-capsule form in RPMI- 1640 medium plus $10 \%$ FBS at $37^{\circ} \mathrm{C}$ with $5 \% \mathrm{CO}_{2}$. Arrows indicate capsule of $\mathrm{C} . \mathrm{g}$-B strain. (B,C) Nuclear protein $\mathbf{( B )}$ and total protein phosphorylation $(\mathbf{C})$ levels in bone marrow-derived macrophages (BMDMs) from wild-type (WT) and Dectin-3(Clec4d)-deficient mice, stimulated with thin- and thick-capsulated C.g-B strain (MOI = 5) for indicated time. (D) Enzyme-linked immune-sorbent assay results for indicated cytokines in WT and Dectin-3-deficient BMDMs, stimulated with thin- and thick-capsulated C.g-B strain $(\mathrm{MOI}=5)$ for $16 \mathrm{~h}$. Data are means \pm SD of triplicate wells and are representative of three independent experiments; NS = no significant difference, ${ }^{\star \star} p<0.01$ and ${ }^{\star \star *} p<0.001$. (E) mRNA expression levels of indicated chemokines in WT and Dectin-3-deficient BMDMs, stimulated with thin- and thick-capsulated C.g-B strain $(\mathrm{MOI}=5)$ for $3 \mathrm{~h}$. 
and specific leukocyte subsets from blood to the site of infection. Here, we found that Dectin-3 deficiency in BMDMs strikingly impaired C.g-B-induced expression of CCL5/RANTES and of C-X-C motif chemokine ligand 9 (CXCL9) and CXCL10 (Figure 1E), which can attract activated $\mathrm{T}$ cells (particularly Th1 cells), DCs, and monocytes by binding to specific CC chemokine receptors (CCR) on these cells, including CCR5 and CXCR3 (28). However, there were slight influences of Dectin-3 deficiency on C.g-B-induced expression of CCL1, CXCL-1, macrophage inflammatory protein- $1 \alpha$, MIP- $1 \beta$, MIP-2, monocyte chemotactic protein-1, granulocyte-macrophage colonystimulating factor (GM-CSF), G-CSF, and M-CSF in BMDMs (Figure 1E). These results suggest that Dectin-3 is also essential for C.g-B-induced NF- $\mathrm{BB}$ and ERK-mediated chemokine productions, resulting in the formation of granulomas and activation of adaptive immunity.

\section{Dectin-3 Can Also Recognize the Capsule of C.n-AD to Induce Pro-Inflammation Responses}

A recent study shows that Dectin-3 is not required for mediating protective immune responses against pulmonary C. $n$-A infection (25). Here, we found that stimulation with C.n-A failed to induce the production of pro-inflammation cytokines, including TNF- $\alpha$, IL-6, IL-12p40, and IL-1 $\beta$ in WT and Dectin-3-deficient BMDMs (Figure 2A). However, stimulation with C.n-AD, C.g$\mathrm{C}$, and C.n-D could induce large amounts of pro-inflammation
A
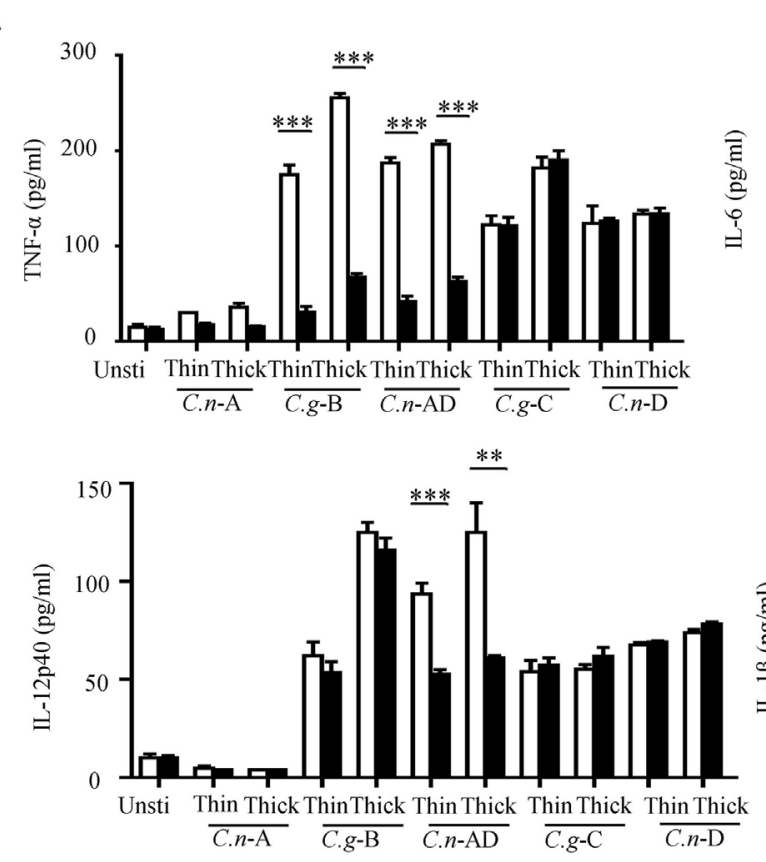

B

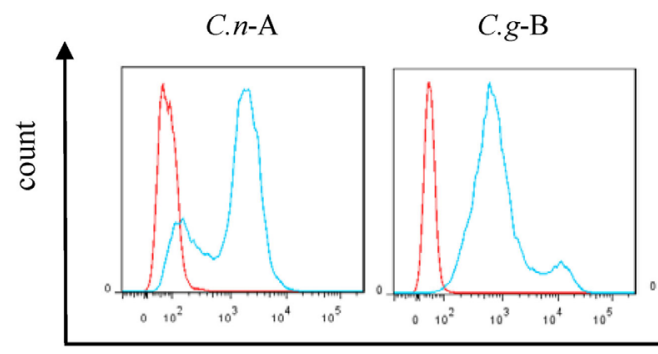

C.g-C
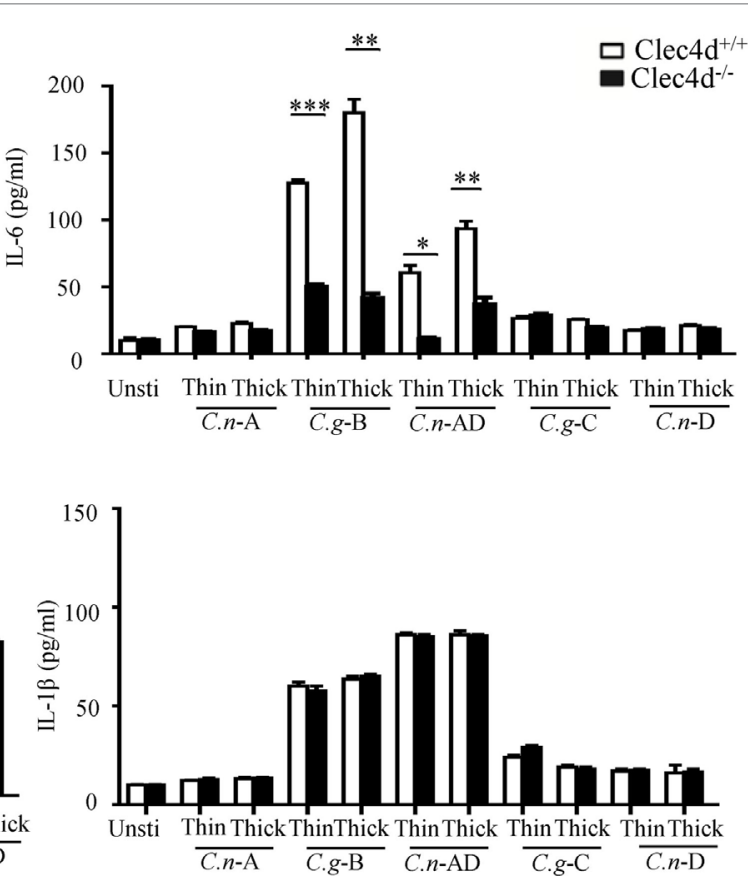

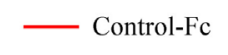

Dectin-3-Fc

C.n-D

C.n-AD
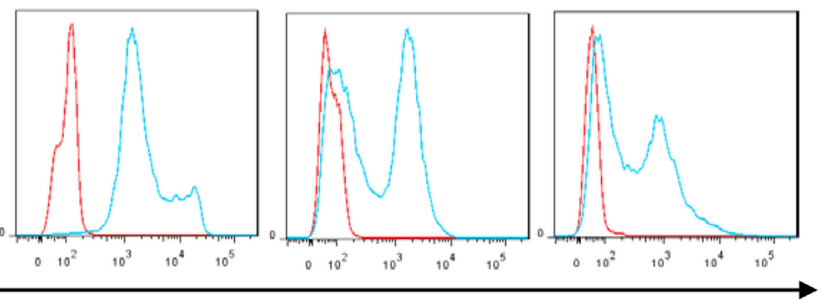

FITC

FIGURE 2 | Dectin-3 is also critical for NF-kB and ERK-mediated pro-inflammation responses induced by Cryptococcus neoformans-AD. (A) Enzyme-linked immune-sorbent assay results for indicated cytokines in wild-type and Dectin-3-deficient bone marrow-derived macrophages, stimulated with thin- and thickcapsulated C.n-A strain H99, C.g-B strain WM179, C.n-AD strain WM628, C.g-C strain NIH312, and C.n-D strain WM629 (MOI = 5) for 16 h. Data are means \pm SD of triplicate wells and are representative of three independent experiments; ${ }^{\star \star} p<0.01$ and ${ }^{\star \star \star} p<0.001$. (B) Fluorescence-activated cell-sorting assay of the binding of human Dectin-3-Fc fusion protein to C.n-A strain H99, C.g-B strain WM179, C.n-AD strain WM628, C.g-C strain NIH312, and C.n-D strain WM629 compared to Control-Fc. 
cytokines, including TNF- $\alpha$, IL-6, and IL-12p40 in WT BMDMs (Figure 2A). Of note, Dectin-3 deficiency significantly impaired the production of TNF- $\alpha$, IL-6, and IL-12p40 in BMDMs when stimulated with both thin- and thick-capsulated C.n-AD, but not C.g-C and C.n-D (Figure 2A). As controls, Dectin-3 deficiency only affected TNF- $\alpha$ and IL- 6 secretion in BMDMs when stimulated with another C.g-B strain WM179 (Figure 2A). Unexpectedly, Dectin-3 deficiency had no influence on the IL-1 $\beta$ production when stimulated with C.n-AD and C.g-B (Figure 2A). These results suggest that Dectin-3 is also critical for mediating pro-inflammation responses induced by C.n-AD, but not C.n-A, C.n-D, or C.g-C.

To explore whether Dectin-3 can bind the surface capsule of Cryptococcus strains, we performed a fluorescence-activated cell-sorting (FACS) screening assay using soluble receptor fusion protein composed of the extracellular portion of Dectin-3 fused with the Fc fragment of human IgG1 antibody (Figure S1 in Supplementary Material). As shown in Figure 2B, Dectin-3-Fc fusion protein could bind all five serotypes of Cryptococcus strains compared to control-Fc. Together, these results indicate that although Dectin-3 can recognize all the serotypes of Cryptococcus strains, only C.n-AD and C.g-B can initiate the pro-inflammation responses dependent of Dectin-3 recognition.

\section{Dectin-3 Directly Recognizes GXM From Cryptococcus Strains}

It has been well-documented that cryptococcal capsule is primarily composed of GXM (29). We extracted extracellular polysaccharides (EPS) from C.n-A, C.g-B, C.g-C, C.n-D, and C. $n$ - $\mathrm{AD}$ as previously reported and the extract procedure was shown in Figure S2A in Supplementary Material. The extracted EPS fraction is a mixture of several polysaccharides, so we performed anion column chromatography to obtain major components for exploring their structural differences. Since the GXMs from Cryptococcus strains contained uronic acid $(32,40)$, we performed reduction reactions to change glucuronic acid (GluA) into glucose (Glu) of EPS for GC-MS analysis (Figure S2B in Supplementary Material; Figure 3A). Monosaccharide composition analysis showed that the EPS from C.n-A, C.g-B, C.g-C, C.n-D, and C.n-AD mainly contained glucose (Glu), xylose (Xyl), and mannose (Man) (Figure S2B in Supplementary Material; Figure 3A), indicating that EPS are primarily composed of GXM, a major cryptococcal capsule component. It has been reported that GXMs from Cryptococcus strains are all comprised of a core repeating unit to which $(1 \rightarrow 2)$-linked and $(1 \rightarrow 4)$-linked $\beta$-D-Xyl units are added in increments of one to four residues (Figure 3B) $(32,40)$. GXMs from C.n-A and C.n-D are mainly substituted with $\mathrm{Xyl}$ at C-2, whereas GXMs from C.g-B, C.g-C, and C.n-AD are substituted at C-2 and at C-4 (Figure 3B). Analytical data showed that molar ratios of Glu/Xyl/Man in C.n-A, C.g-B, C.g-C, C.n-D, and C.n-AD were calculated as 0.67:2:3, 0.6:3:3, 0.5:4:3, 0.55:1:3, and 0.58:2:3, respectively (Figure S2C in Supplementary Material; Figure 3C), which demonstrated that all serotypes of GXM have the same repeating unit composed of mannan and GluA as main chain whereas the quantity and position of xylose residue is the major structural difference of GXMs among different serotypes.
To determine whether Dectin-3 can directly recognize GXM from Cryptococcus strains, we used an enzyme-linked immunesorbent assay (ELISA)-based method to examine the direct binding of plate-coated GXM extracted from Cryptococcus strains with Fc-fusion Dectin-3 protein, which were known to be bond with plate-coated $\alpha-1,2$-mannan. We found that Dectin-3 could bind GXM from all five serotypes of Cryptococcus strains (Figure 3D). Furthermore, to determine which group does Dectin-3 directly recognizes, we used xylose, mannan, and GluA, to saturate Dectin-3-Fc fusion protein separately before ELISA binding assay. It showed that both mannan and GluA instead of xylose saturation could block the binding of Dectin-3 to GXM (Figure 3D). Together, these data indicate that Dectin-3 can directly recognize GXM from all five serotypes of Cryptococcus strains. The general repeating unit composed of mannan and GluA determines its binding with Dectin-3.

\section{Dectin-3 Recognizes GXM From C.g-B and C.n-AD to Induce NF-kB- and ERK- Mediated Pro-Inflammation Responses}

To further determine whether Dectin-3 recognizes GXM from C.g-B and C.n-AD to elicit pro-inflammation responses, we stimulated WT and Dectin-3-deficent BMDMs with plate-coated GXM extracted from C.g-B (GXM-B) and C.n-AD (GXM-AD). We found that stimulation with plate-coated GXM-B and GXM-AD induced I $\kappa \mathrm{B} \alpha$ phosphorylation and degradation and ERK phosphorylation (Figures 4A,B). In contrast, Dectin-3 deficiency in BMDMs strikingly impaired GXM-B or GXM-AD induced activation of NF- $\kappa B$ and ERK pathways (Figures $4 A, B$ ). Furthermore, we found that only stimulation with plate-coated GXM-B and GXM-AD potently induced the secretion of proinflammation cytokines, including TNF- $\alpha$, IL-6, and IL-12p40, whereas Dectin-3 deficiency significantly impaired these responses (Figure 4C). We further examined the effect of inhibiting NF- $\kappa \mathrm{B}$ or ERK signaling on pro-inflammation cytokine production by WT BMDMs. We found that treatment with TPCA, a specific inhibitor for NF- $\kappa \mathrm{B}$ activation, significantly suppressed TNF $\alpha$ and IL- 6 production by WT BMDMs when stimulated with plate-coated GXM extracted from either C.g-B or C.n-AD (Figures 4D,E). However, treatment with U0126, an inhibitor for ERK activation, only blocked TNF $\alpha$ production and had no inhibitory effect on GXM-induced IL-6 production (Figures 4D,E). To verify the pro-inflammatory response to GXM from EPS, we also used capsular polysaccharides (CPS) from five serotypes of Cryptococcus strains to stimulate BMDMs. It was shown that the production of IL- 6 and TNF- $\alpha$ induced by CPS-B and CPS-AD was dependent of Dectin-3 recognition, which was consistent with the effects of GXM from EPS (Figure 4F). Thus, these data suggest that Dectin-3 recognizes GXM from C.g-B and C.n-AD to trigger NF- $\mathrm{KB}-$ and ERK-mediated proinflammation responses.

\section{Dectin-3-Deficient Mice Are Highly Susceptible to Pulmonary Infection With C.g-B and C.n-AD}

To provide genetic evidence that Dectin-3 plays a critical role against experimental pulmonary cryptococcosis, we explored 
A
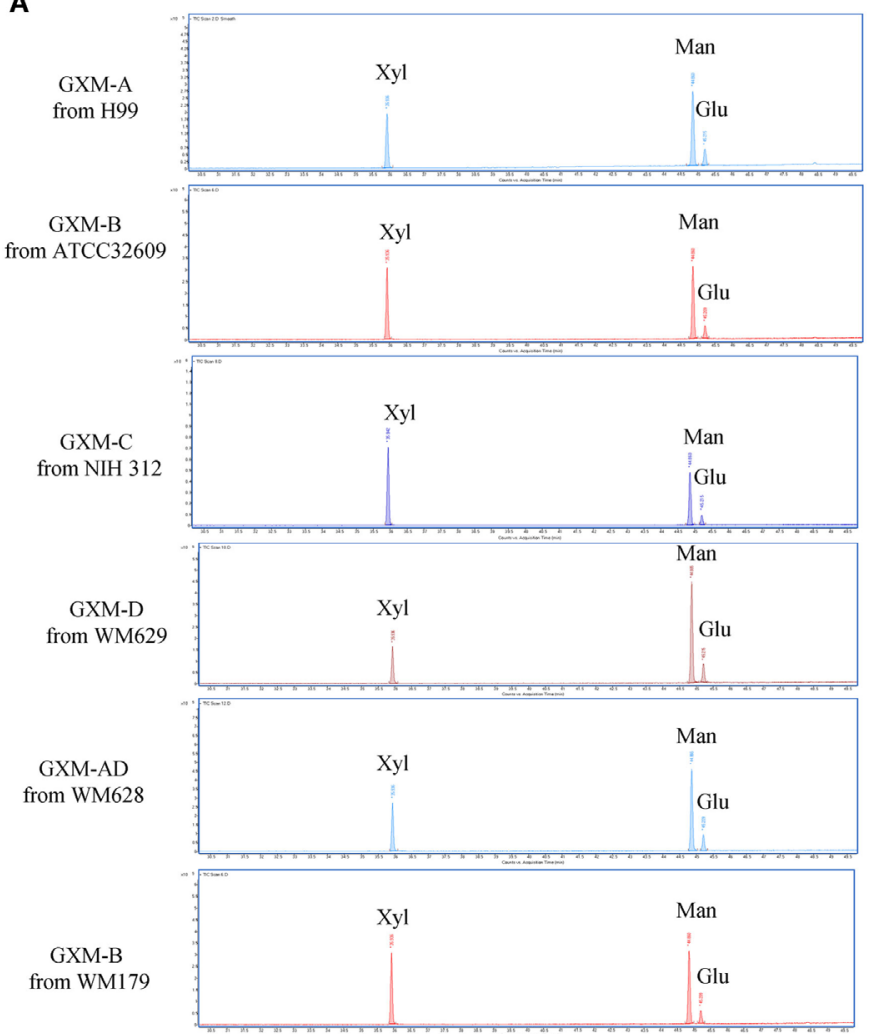

Structure groups

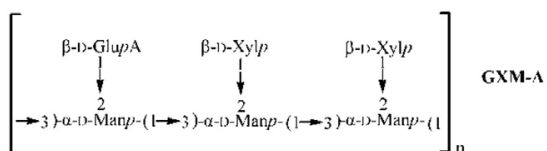

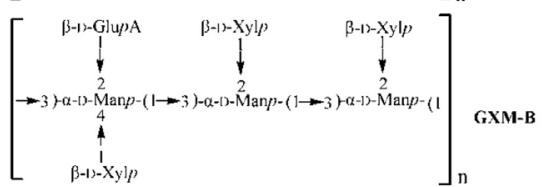

(n)
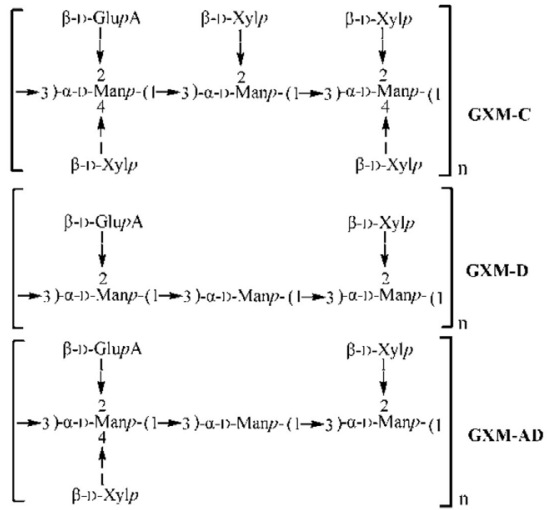

C

\begin{tabular}{ccccc}
\hline Strain & Serotype & Glucose & Xylose & Mannose \\
\hline H99 & A & 0.67 & 2 & 3 \\
ATCC32609 & B & 0.6 & 3 & 3 \\
WM179 & B & 0.5 & 3 & 3 \\
NIH312 & C & 0.5 & 4 & 3 \\
WM629 & D & 0.55 & 1 & 3 \\
WM628 & AD & 0.6 & 2 & 3 \\
\hline
\end{tabular}

D
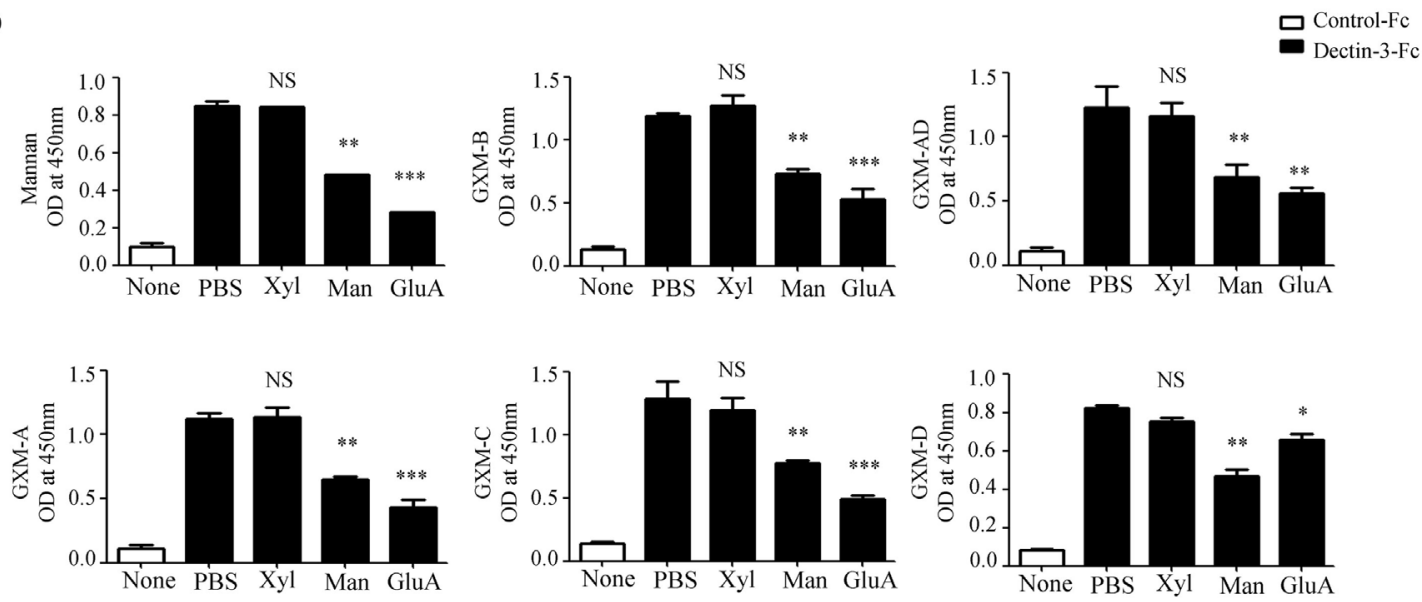

FIGURE 3 | Dectin-3 directly recognizes GXM from Cryptococcus strains. (A) Monosaccharide assay of GXM from C.n-A strain H99, C.g-B strain ATCC32609, C.g-B strain WM179, C.n-AD strain WM628, C.g-C strain NIH312, and C.n-D strain WM629 by gas chromatography-mass spectrometry (GC-MS) after uronic acid reduction treatment. (B) Predicted structures of GXM from five serotypes of Cryptococcus strains. (C) Analysis of monosaccharide composition of GXM. (D) Enzyme-linked immune-sorbent assay binding assay of human control-Fc and Dectin-3-Fc fusion protein to five serotypes of GXM and mannan. Dectin-3-FC fusion protein was saturated with mannan/xylose/glucuronic acid, respectively. Data are means \pm SD of triplicate wells and are representative of three independent experiments; NS = no significant difference, ${ }^{*} p<0.05,{ }^{* \star} p<0.01$, and ${ }^{\star * *} p<0.001$. 

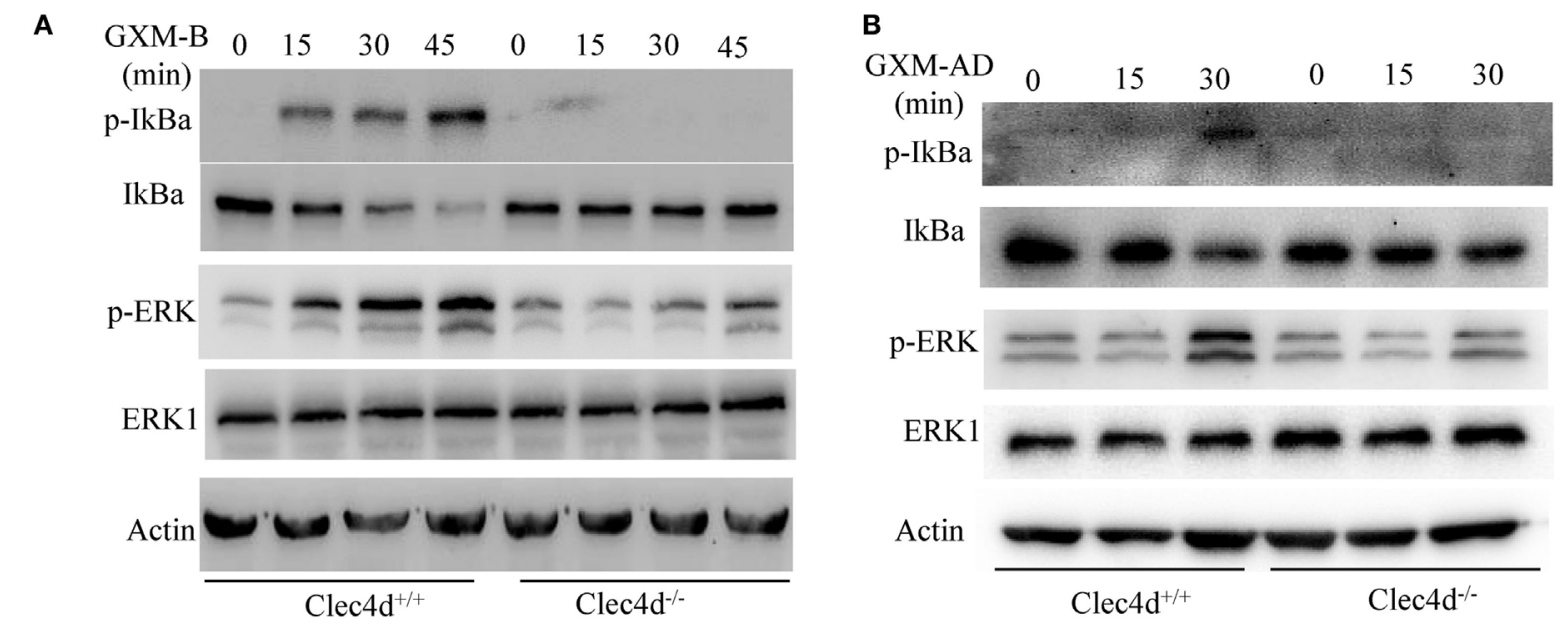

C
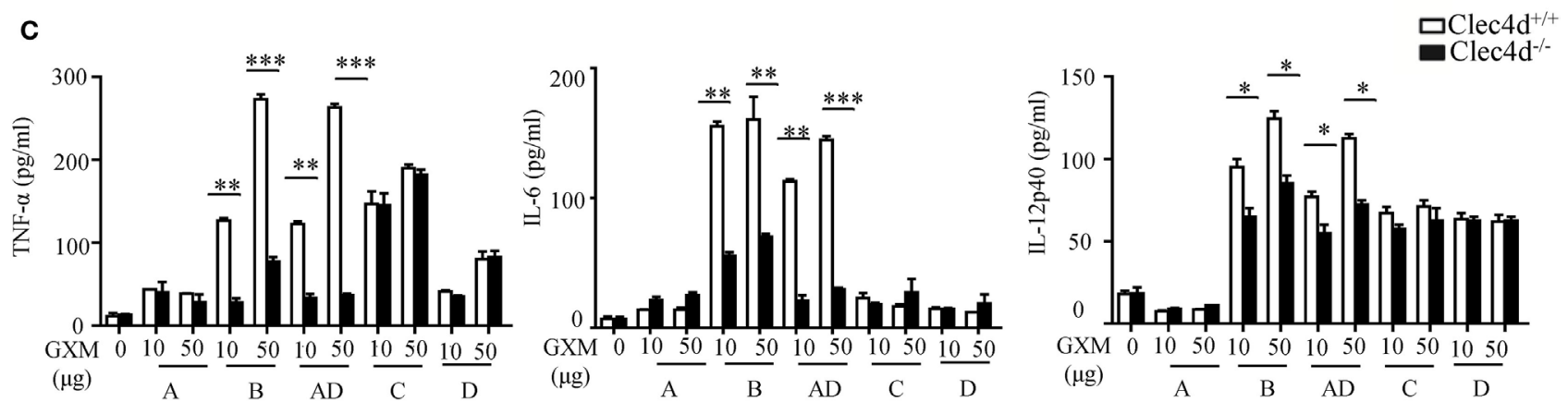

D
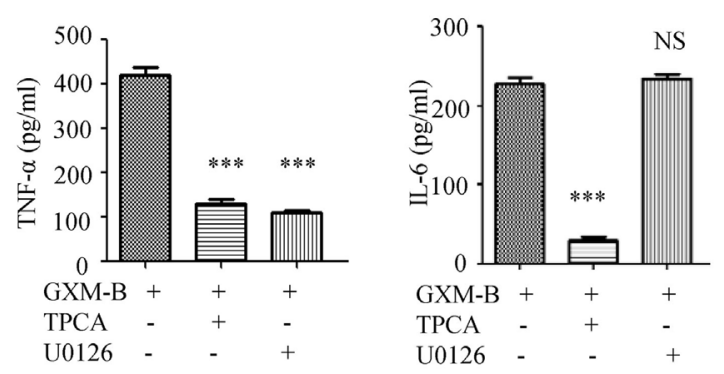

E

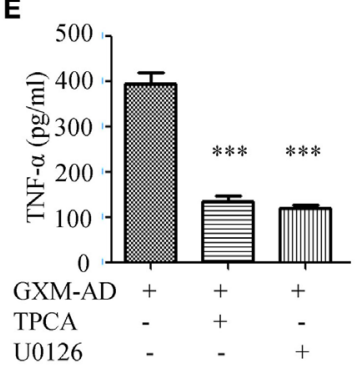

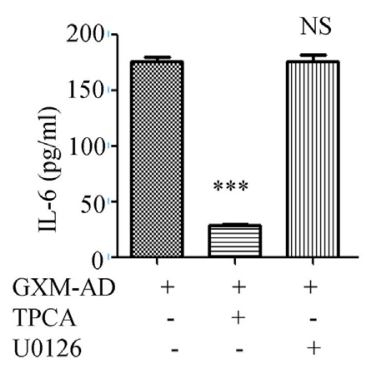

F
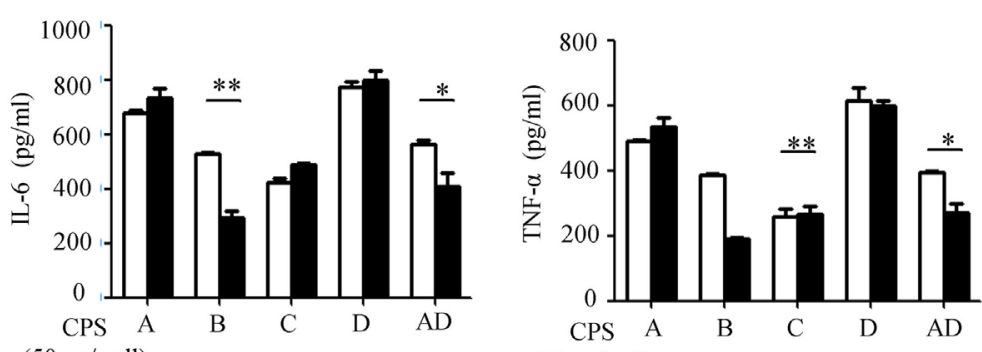

(50 $\mu \mathrm{g} / \mathrm{well})$

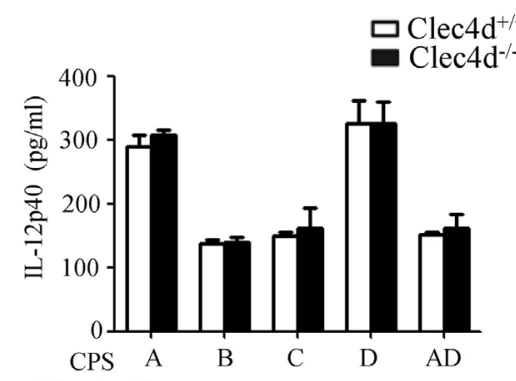

FIGURE 4 | GXMs from Cryptococcus gattii-B and Cryptococcus neoformans-AD induce NF-kB- and ERK-mediated pro-inflammation responses through Dectin-3. (A,B) Protein phosphorylation levels in bone marrow-derived macrophages (BMDMs) from wild-type (WT) and Dectin-3 (Clec4d)-deficient mice, stimulated with plate-coated $50 \mu \mathrm{g} /$ well GXM from C.g-B strain ATCC32609 [GXM-B, (A)] or C.n-AD strain WM628 [GXM-AD, (B)] for indicated time. (C) Enzyme-linked immunesorbent assay (ELISA) results for indicated cytokines in WT and Dectin-3-deficient BMDMs, stimulated with plate-coated GXM extracted from C.n-A strain H99, C.g-B strain ATCC32609, C.n-AD strain WM628, C.g-C strain NIH312, and C.n-D strain WM629 for 16 h. (D,E) ELISA results for indicated cytokines in WT BMDMs, pretreated with $10 \mu \mathrm{M}$ TPCA (p65 inhibitor) or $10 \mu \mathrm{M}$ U0126 (ERK inhibitor) and stimulated with plate-Coated GXM-B (D) or GXM-AD (E) for 16 h. (F) ELISA results for indicated cytokines in WT and Dectin-3-deficient BMDMs, stimulated with plate-coated CPS (50 $\mu \mathrm{g} /$ well) of five serotypes for 16 h. Data are means \pm SD of triplicate wells and are representative of three independent experiments; NS $=$ no significant difference, ${ }^{\star} p<0.05$, ${ }^{* \star} p<0.01$, and ${ }^{\star \star \star} p<0.001$. 
the overall impact of Dectin-3 deficiency during an experimental pulmonary infection with C.g-B and C.n-AD in mice. WT and Dectin-3-deficient mice received an intratracheal inoculation with C.g-B strain ATCC32609. Survival was monitored for greater than 60 days post-inoculation, while pulmonary fungal burden was evaluated in a separate group of infected mice at select time points post-inoculation (Figures $5 \mathbf{A}, \mathbf{B}$ ). We found that all infected Dectin-3-deficient mice died during 45 days, whereas about $70 \%$ infected WT mice survived for more than 65 days $(p<0.01$, Figure 5A). Consistently, the burdens of C.g-B in lung and brain on day 3 after infection were significantly higher in Dectin-3-deficient mice than in WT mice $(p<0.01$ and $p<0.001$, respectively, Figure 5B). We then conducted a histological analysis of the lungs on day 14 after infection. Massive multiplication of yeast cells with poor granulomatous responses was observed in the alveolar spaces of Dectin-3-deficient mice, whereas WT mice showed a seldom number of yeast cells, which were mostly encapsulated in the granulomatous tissues (Figure 5C). We also detected the significant reductions of pro-inflammatory cytokine including TNF- $\alpha$ and IL- 6 in the lungs of Dectin-3-deficient mice than those in WT mice (Figure 5D). However, the amount of IL-12p40 and IL-1 $\beta$ was not significantly decreased in the lungs of Dectin-3-deficient mice (Figure 5D). These results indicate that Dectin-3-mediated signaling is involved in the elimination of pulmonary C. $g$-B infection and induction of a protective inflammatory response.

Consistent with the results in the previous study (25), WT and Dectin-3-deficient mice showed an equivalent susceptibility to pulmonary C.n-A strain H99 infection (Figure 5E). Also, no significant differences were observed in the pulmonary fungal burdens of Dectin-3-deficient mice compared to WT mice on day 3 after infection with C.n-A strain H99 (Figure 5F). These results indicate that Dectin-3 is not required for host defense against pulmonary C.n-A infection. Moreover, Dectin-3-deficient mice exhibited higher fungal burdens in lungs than WT mice after intratracheal infection with C.n-AD strain WM628 and C.g-B strain WM179 ( $p<0.01$, Figure 5G). However, there were no differences in lung fungal burdens between WT and Dectin3-deficient mice when infected with C.g-C strain NIH312 and C.n-D strain WM629 (Figure 5G). Together, these results demonstrate that Dectin-3-deficient mice are highly susceptible to pulmonary C.g-B and C.n-AD infections.

\section{Dectin-3 Is Critical for Activation of AMs After Pulmonary C.g-B and C.n-AD Infection}

It has been well-documented that AMs constitute the first line of host defense against pulmonary Cryptococcus infections and the subsequent inflammatory response, resulting in an influx of neutrophils and monocytes, affords a second line of defense (41). To explore whether Dectin-3 is required for AM and neutrophil accumulation at the early stage of pulmonary infection with C.g-B and C.n-AD, we determined cellular composition of lungs and found that infected WT mice with C.g-B and C.n-AD on day 1 showed a prominent AM-biased response, while Dectin-3 deficiency significantly impaired AM accumulation in lungs $(p<0.001$, Figure 6A; Figure S3A in Supplementary Material). However, either pulmonary Cryptococcus infection or Dectin-3 deficiency had no influences on neutrophil accumulation in lungs at the early stage (Figure 6B; Figure S3B in Supplementary Material). Furthermore, we found that AMs sorted from naïve WT mice had high killing activities against C.n-A, C.n-AD, and C.g-B in a dose-dependent manner, whereas Dectin-3 deficiency significantly reduced killing activities of AMs against C.n-AD and C.g-B, but not C.n-A (Figure 6C). These data suggest that Dectin-3 controls AM accumulation and activities after pulmonary infection with C.g-B and C.n-AD.

To further examine roles of Dectin-3 in AM-mediated host defense against pulmonary infection with C.g-B, we performed an adoptive transferring of equal numbers of AMs between WT and Dectin-3-deficient (KO) mice (Figure S3C in Supplementary Material). We found that transferred AMs sorted from WT mice into KO mice could significantly increase AM accumulation and protein levels of pro-inflammation cytokines, including TNF- $\alpha$, IL-6, and IL-12p40 in lung on day 1 after pulmonary infection with C.g-B (Figures 6D,F). Furthermore, the transfer of WT AMs into $\mathrm{KO}$ mice significantly reduced fungal burdens in lungs after intratracheal infection with C.g-B ( $p<0.01$, Figure 6G). In contrast, transferred AMs sorted from $\mathrm{KO}$ mice into $\mathrm{KO}$ mice had no any influences on AM accumulation, but significantly increased neutrophil accumulation in lungs (Figures 6D,E). These data confirm that Dectin-3 is critical for accumulation and activities of AM against pulmonary C.g-B infection.

\section{CARD9 Is Critical for Pro-Inflammation Responses Induced by GXMs}

It has been shown that CARD9 operates downstream of CLRs for activating NF- $\mathrm{KB}$ and ERK pathways $(26,27)$. To explore whether CARD9 is required for C.g-B-induced pro-inflammation responses, we stimulated BMDMs from WT and CARD9-deficient mice with plate-coated GXM extracted from C.g-B (GXM-B), and found that CARD9 deficiency in BMDMs completely impaired GXM-B induced activation of NF- $\kappa \mathrm{B}$ and ERK pathways (Figures S4A,B in Supplementary Material). Furthermore, we found that CARD9 deficiency in BMDMs significantly impaired GXM-B induced secretion of pro-inflammation cytokines, including TNF$\alpha$ and IL-6, but not IL12-p40 and IL-1 $\beta$ (Figure 7A). Moreover, CARD9 deficiency in BMDMs also significantly impaired the production of TNF- $\alpha$ and IL- 6 when stimulated with platecoated GXM extracted from C.n-AD, C.g-C, and C.n-D, but not C.n-A (Figure 7A). These data suggest that CARD9 is essential for $\mathrm{NF}-\kappa \mathrm{B}$ and ERK-mediated pro-inflammation responses induced by GXM from C.g-B, C.g-C, C.n-D, and C.n-AD.

To examine the effect of CARD9 deficiency on the clinical course of cryptococcal infection, we intratracheally infected WT and CARD9-deficient mice with different Cryptococcus strains. We found that the burdens in lung and brain were significantly higher in CARD9-deficient mice than those in WT mice on day 3 and 14 after infection with C.g-B strain ATCC32609 (Figure 7B; Figure S4C in Supplementary Material). Furthermore, the histological analysis of the lungs on day 14 after infection showed massive multiplication of yeast cells with poor granulomatous 
A

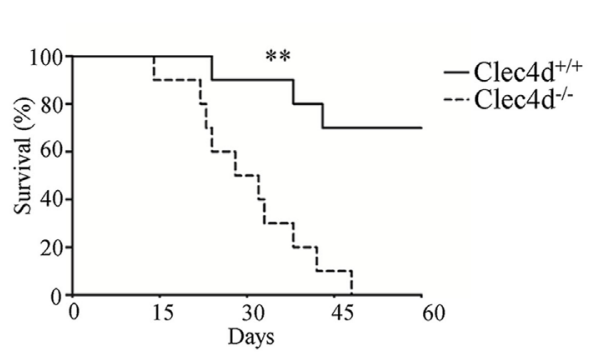

C

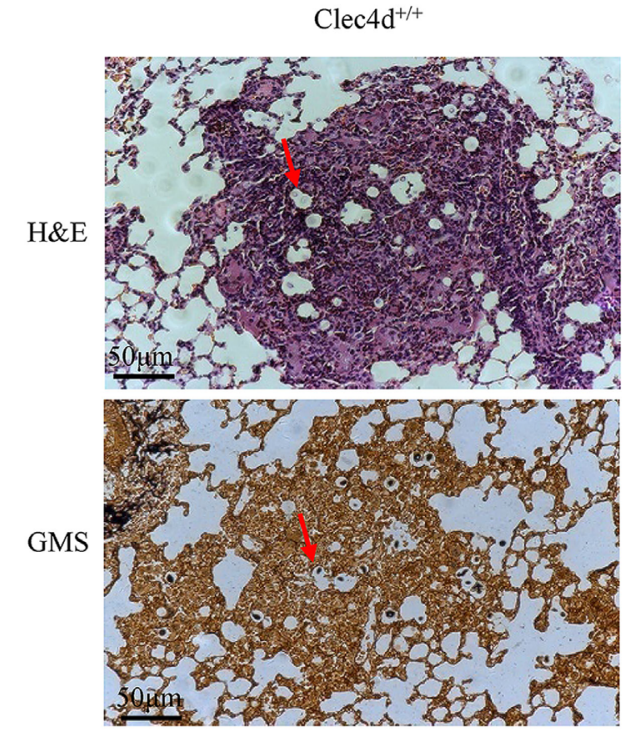

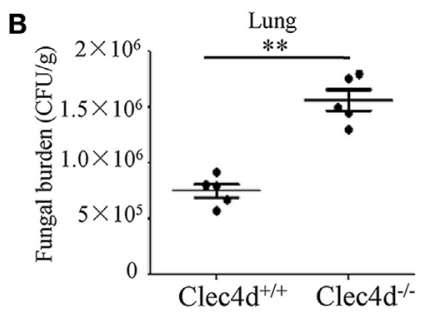

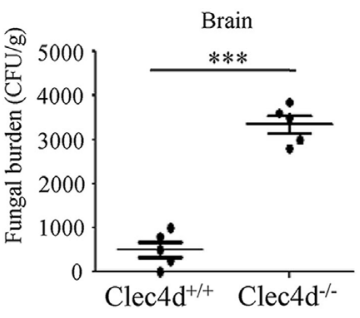

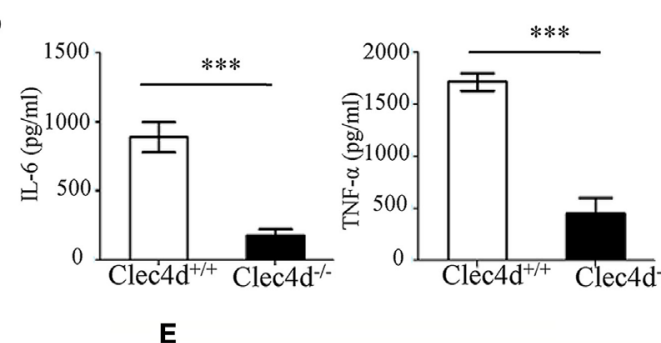

E

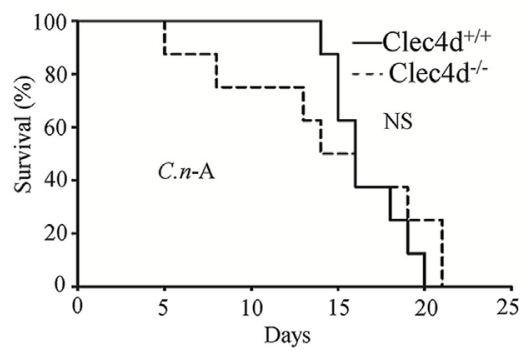

Clec $4 \mathrm{~d}^{-/-}$

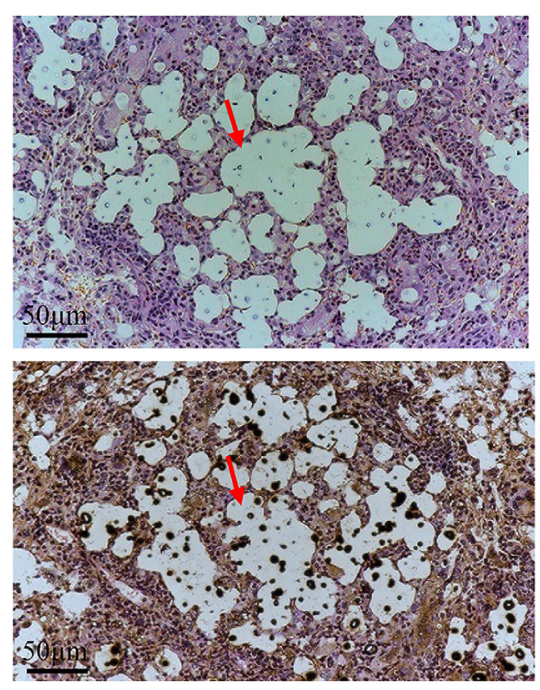

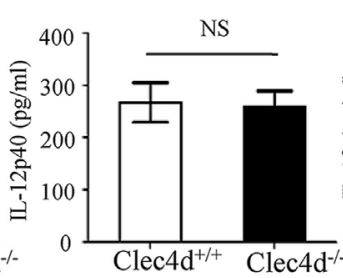

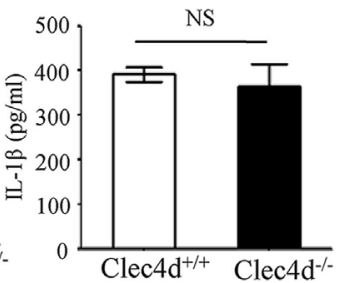

$\mathbf{F}$

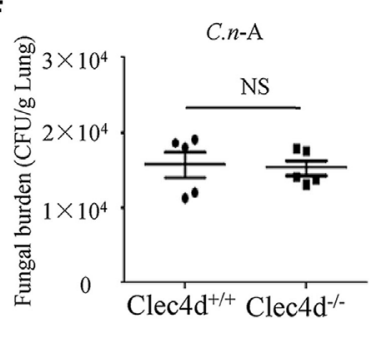

G
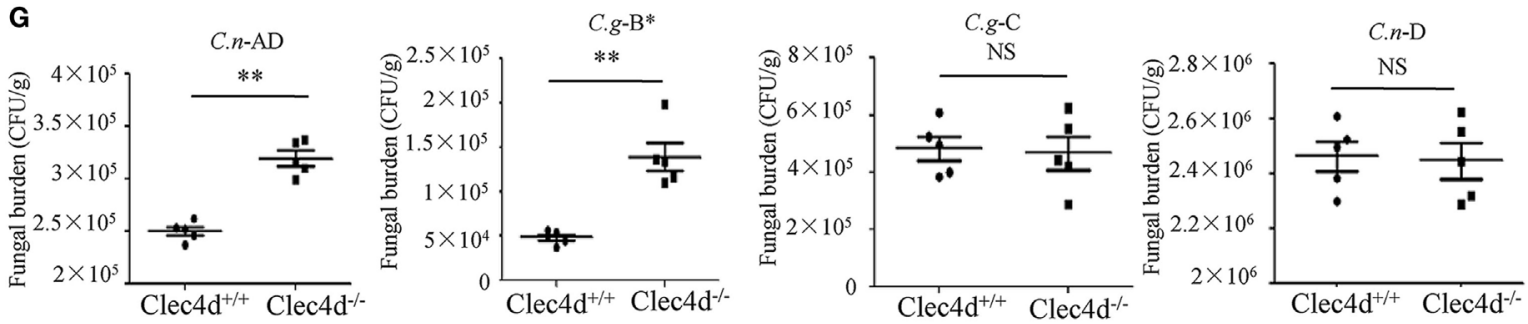

FIGURE 5 | Continued 
FIGURE 5 | Dectin-3-deficient-mice are highly susceptible to pulmonary infection with Cryptococcus gattii-B and Cryptococcus neoformans-AD. (A) Survival curves of wild-type (WT) and Dectin-3-deficient (Clec4d) mice ( $n=10$ for each group) after intratracheal infection with $1 \times 10^{6}$ CFU of . $g$-B strain ATCC32609. (B) CFU assays of lung and brain of WT and Dectin-3-deficient mice infected intratracheally with $1 \times 10^{5} \mathrm{CFU}$ of C.g-B on day 3 after infection. (C) Histopathology was analyzed with hematoxylin and eosin (H\&E) and Gomori's methenamine silver staining on day 14 after intratracheal infection with $1 \times 10^{6} \mathrm{CFU}$ of $\mathrm{C}$. $g$-B. (D) Enzyme-linked immune-sorbent assay results of TNF-a, IL-6, IL-12p40, and IL-1 $\beta$ in WT and Dectin-3-deficient mice lung homogenate on day 1 after intratracheal infection with $1 \times 10^{5} \mathrm{CFU}$ of C.g-B. (E) Survival curves of WT and Dectin-3-deficient mice $(n=10$ for each group) after intratracheal infection with $1 \times 10^{6}$ CFU of C.n-A strain H99. (F) Lung CFU assay of WT and Dectin-3-deficient mice on day 3 after infection with $1 \times 10^{5}$ CFU of C.n-A. (G) Lung CFU assays of WT and Dectin-3-deficient mice on day 3 after intratracheal infection with $1 \times 10^{5}$ CFU of C.n-AD strain WM628, C.g-B strain WM179, C.g-C strain NIH312, or C.n-D strain WM629, respectively. Data are means \pm SD of triplicate wells and are representative of three independent experiments; ${ }^{* *} p<0.01$ and ${ }^{* \star *} p<0.001$.
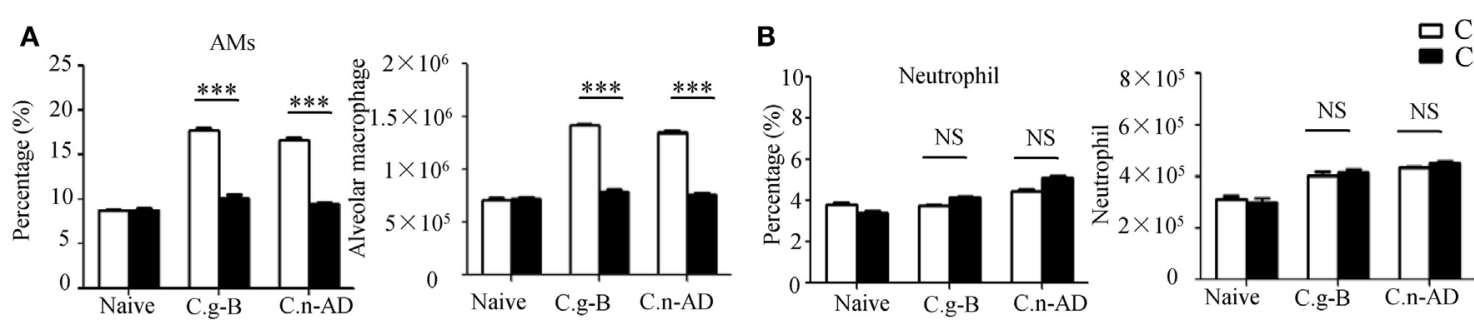

C
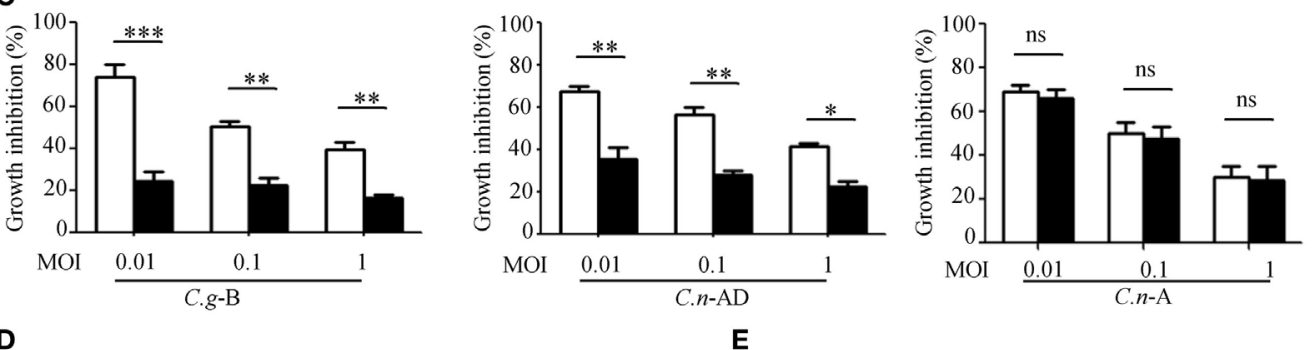

D
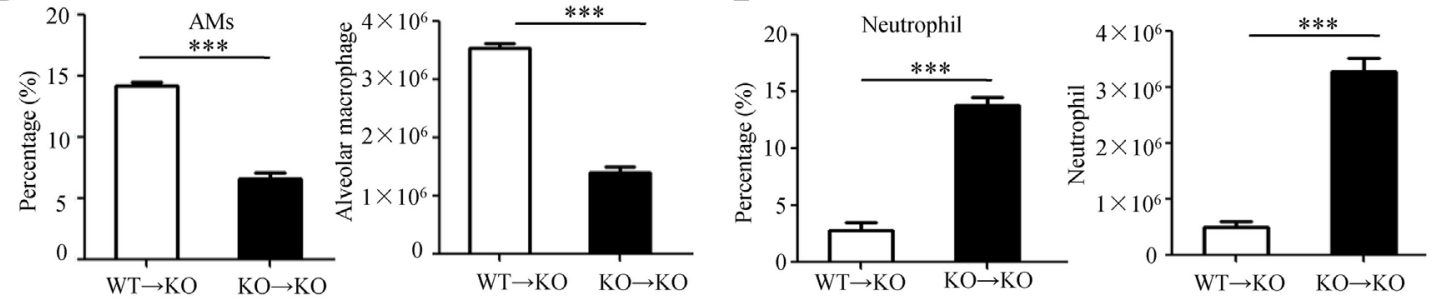

$\mathbf{F}$
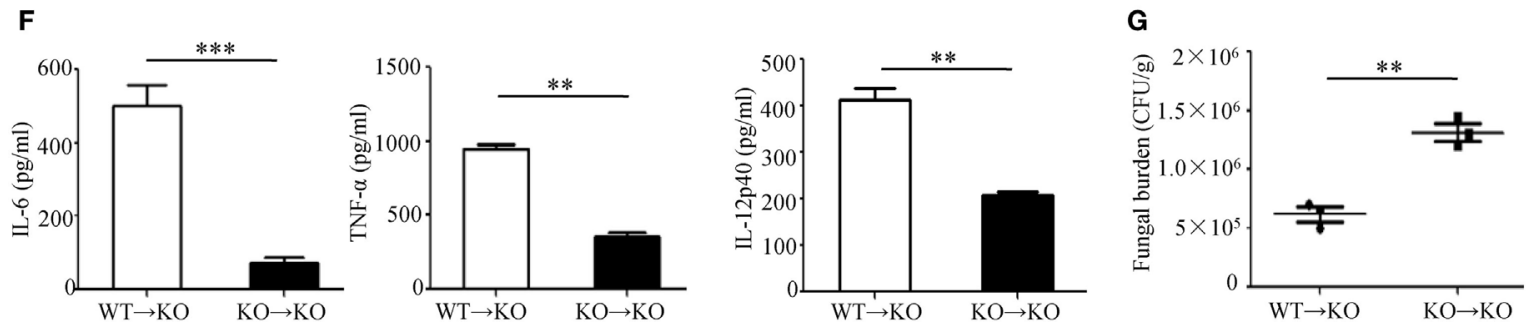

FIGURE 6 | Dectin-3 is critical for activation of alveolar macrophages (AMs) after pulmonary Cryptococcus gattii-B and Cryptococcus neoformans-AD infection. (A,B) AM [CD11 $\mathrm{C}^{+}$SiglecF $\left.{ }^{+}, \mathbf{( A )}\right]$ and neutrophil $\left[\mathrm{CD} 11 \mathrm{~b}^{+} \mathrm{Ly}_{6 \mathrm{G}}{ }^{+}, \mathbf{( B )}\right]$ counts in lungs of wild-type (WT) and Dectin-3-deficient mice on day 1 after intratracheal infection with C.g-B strain ATCC32609 or C.n-AD strain WM628. (C) Killing activity of AM sorted from lungs of WT and Dectin-3-deficient mice. C.g-B strain ATCC32609, C.n-AD strain WM628, or C.n-A strain H99 (MOI = 0.01, 0.1, and 1) was co-cultured with or without sorted AM for 6 h and the growth inhibition by $\mathrm{AM}$ was calculated. (D,E) AM [CD11 $\mathrm{C}^{+}$SiglecF+, (D)] and neutrophil [CD11b+Ly6G+ $\mathrm{C}^{+}$(E)] counts in lungs of Dectin-3-deficient mice, which received intravenous adopt transfer of AMs $\left(5 \times 10^{5}\right.$ cells/mouse) sorted from WT or Dectin-3-deficient mice before infected with $1 \times 10^{5}$ CFU of C.g-B strain ATCC32609. (F) ELISA results of TNF-a, IL-6, and IL-12p40 in lung homogenate of Dectin-3-deficient mice, receiving intravenous adopt transfer of AM, on day 1 post infection. (G) Lung CFU assays of Dectin-3-deficient mice, receiving intravenous adopt transfer of $\mathrm{AM}$, on day 3 post infection. ${ }^{*} p<0.05$, ${ }^{\star *} p<0.01$, and ${ }^{* \star *} p<0.001$.

responses in the alveolar spaces of CARD9-deficient mice (Figure 7C), which is consistent with the findings observed in infected Dectin-3-deficient mice with C.g-B strain ATCC32609
(Figure 5C). Moreover, CARD9 deficiency completely blocked AM accumulation (Figure 7D; Figure S4D in Supplementary Material) and significantly reduced secretion of pro-inflammatory 
cytokine including TNF- $\alpha$ and IL- 6 in the lungs on day 1 after infection with C.g-B (Figure 7E). Unexpectedly, CARD9 deficiency slightly increased neutrophil accumulation in the lungs (Figure 7D; Figure S4E in Supplementary Material), which has been reported in a previous study (28). Moreover, CARD9deficient mice exhibited higher fungal burdens in lungs than WT mice after intratracheal infection with C.n-AD strain WM628, C.g-C strain NIH312, and C.n-D strain WM629 for 3 days

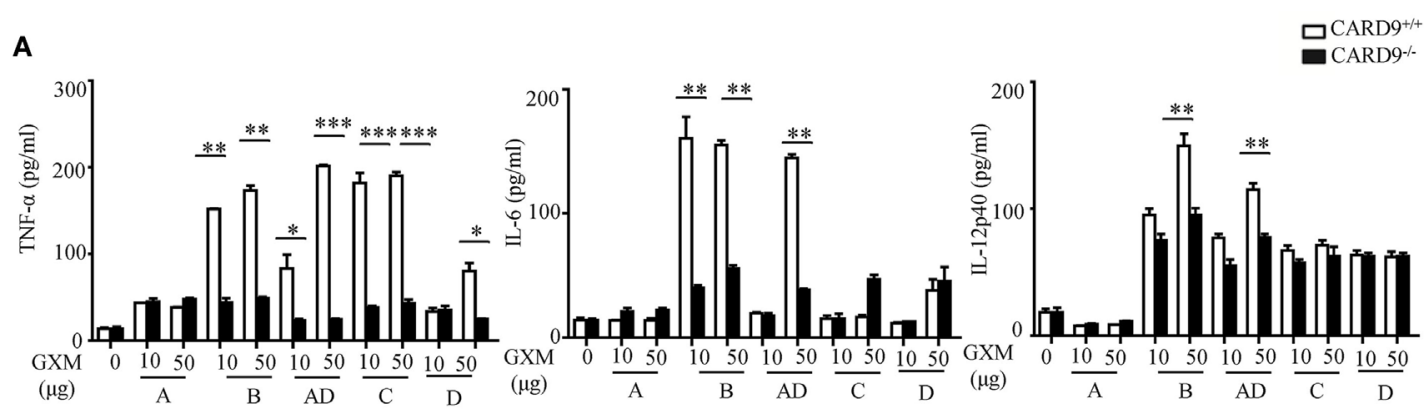

B
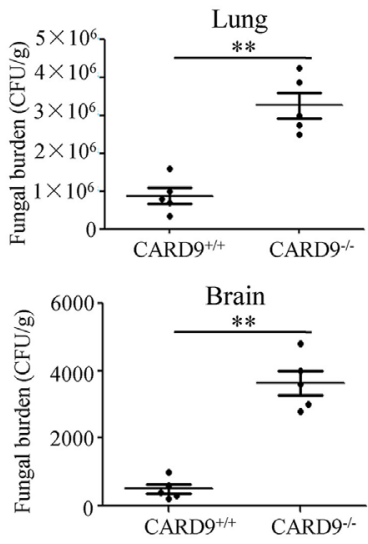

D

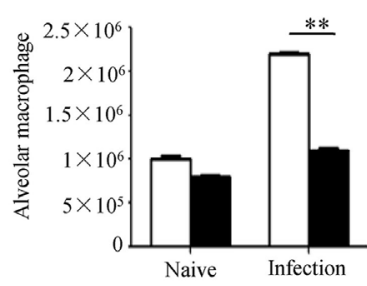

$\mathbf{F}$

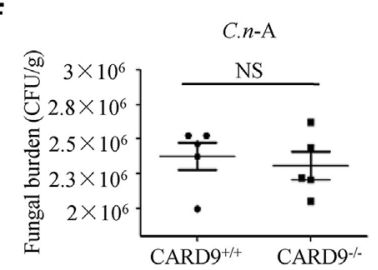

G
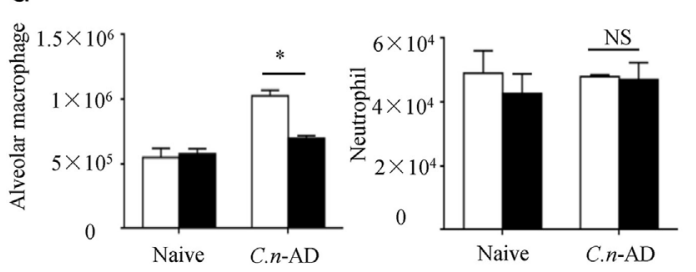

C
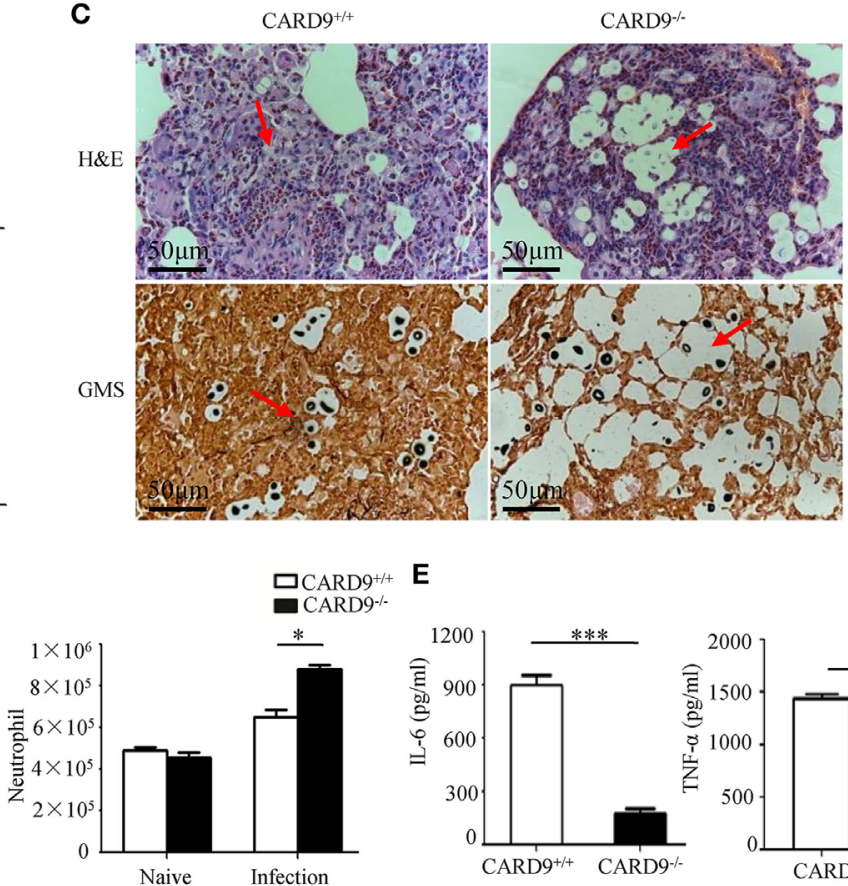

E

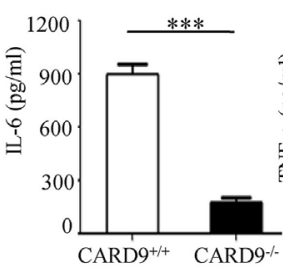

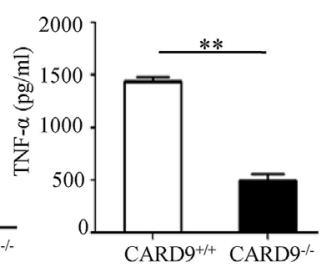
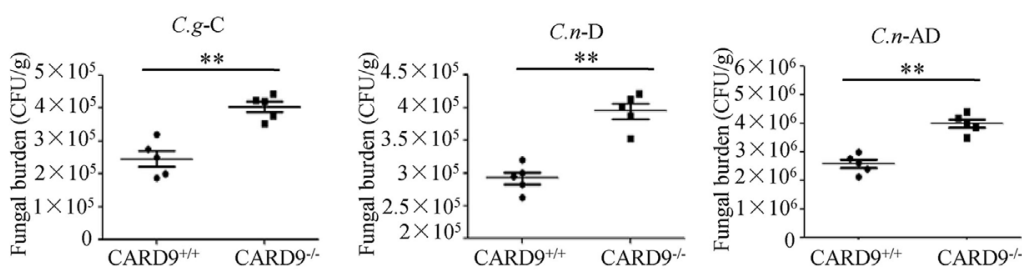

H

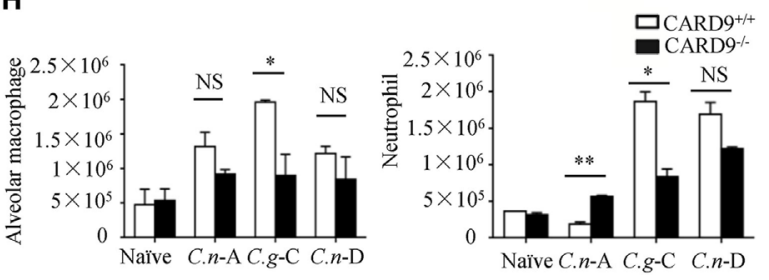

FIGURE 7 | Continued 
FIGURE 7 | Caspase recruitment domain family member 9 (CARD9) is critical for pro-inflammation responses induced by GXMs. (A) Enzyme-linked immune-sorbent assay (ELISA) results for indicated cytokines in wild-type (WT) and CARD9-deficient bone marrow-derived macrophages (BMDMs), stimulated with plate-coated GXM extracted from C.n-A strain H99, C.g-B strain ATCC32609, C.n-AD strain WM628, C.g-C strain NIH312, and C.n-D strain WM629 for 16 h. (B) CFU assays of lung and brain of WT and CARD9-deficient mice after intratracheal infection with $1 \times 10^{5}$ CFU of C.g-B strain ATCC32609 on day 3 post infection. (C) Histopathology with hematoxylin \& eosin and Gomori's methenamine silver staining on day 14 after intratracheal infection with $1 \times 10^{6}$ CFU of C.g-B strain ATCC32609. (D) Alveolar macrophages (AMs) $\left(C D 11 \mathrm{c}^{+}\right.$SiglecF+ $\left.{ }^{+}\right)$and neutrophil $\left(C D 11 \mathrm{~b}^{+} \mathrm{Ly}_{6 \mathrm{G}} \mathrm{G}^{+}\right.$) counts in lungs of WT and CARD9-deficient mice on day 1 after intratracheal infection with C.g-B strain ATCC32609. (E) ELISA results of TNF-a and IL-6 in lung homogenate of WT and CARD9-deficient mice on day 1 after infection. (F) Lung CFU assays of WT and CARD9-deficient mice on day 3 after intratracheal infection with $1 \times 10^{5}$ CFU of C.n-AD strain WM628, C.g-B strain WM179, C.g-C strain NIH312, or C.n-D strain WM629, respectively. (G,H) AM (CD11 $\mathrm{C}^{+}$SiglecF+) and neutrophil (CD11b+Ly6G+) counts in lungs of WT and CARD9-deficient mice on day 1 after intratracheal infection with C.n-AD strain WM628 (G) or C.n-A strain H99, C.g-C strain NIH312, and C.n-D strain WM629 (H). Data are means \pm SD of triplicate wells and are representative of three independent experiments; ${ }^{*} p<0.05,{ }^{* *} p<0.01$, and ${ }^{* \star *} p<0.001$.

$(p<0.01$, Figure 7F). However, there were no differences in lung fungal burdens between WT- and CARD9-deficient mice when infected with C.n-A strain H99 on day 3 (Figure 7F). Moreover, CARD9 deficiency completely blocked AM accumulation in the lungs at day 1 after infection with C.n-AD strain WM628 and C.g-C strain NIH312 (Figures 7G,H; Figures S5 and S6 in Supplementary Material). Unexpectedly, CARD9 deficiency had no influences on AM accumulation in the lungs at day 1 after infection with C.n-D strain WM629 (Figure 7H; Figure S6 in Supplementary Material). These data show that CARD9 is critical for accumulation and activities of AM against pulmonary infection with C.g-B, C.g-C, and C.n-AD.

\section{DISCUSSION}

Germline encoded PRRs recognize a variety of microbial moieties which, once engaged, result in the activation of antimicrobial host defense and stimulation of adaptive immune responses. The role of CLRs during cryptococcosis is of interest as recent studies have defined their role in the recognition of carbohydrate moieties and host defense against other fungal pathogens $(15,16,18,21,23,42)$. In this study, we have extensively characterized the role of Dectin-3 and CARD9 during pulmonary cryptococcosis using an experimental murine model of pulmonary infection with C. neoformans and C. gattii. Upon pulmonary C.n-AD and C.g-B infection, Dectin-3- and CARD9deficient mice were highly susceptible and showed augmented lung injury due to impairment of AM accumulation and killing activities, and subsequent productions of pro-inflammation cytokines and chemokines. More importantly, we showed that Dectin-3 directly recognized GXMs from C.n-AD and C.g-B to activate CARD9-mediated NF- $\mathrm{BB}$ and ERK pathways for initiating anti-fungal host responses against $C$. neoformans and C. gattii. Thus, our study provides the first biological and genetic evidence demonstrating that Dectin-3 recognizes GXM of C. neoformans serotype $\mathrm{AD}$ and C. gattii serotype B to initiate host defense against cryptococcosis.

However, there are still some intriguing parts in our study that worth further exploration. There has been over 120 years since the first isolation of Cryptococcus (43). Over several decades, Cryptococcus has been classified into two varieties including five serotypes: C. neoformans for serotypes A, D, and $\mathrm{AD}$, and C. gattii for serotypes B and C (44). Furthermore, with the rapid development of PCR and DNA sequencing, these species have been further sub-divided into several genotypes: VNI,
VII, VNB, VNIV, and VGI-IV (45). In this study, we used two different strains of C. gattii serotype B (ATCC32609 and WM179). Although they are the same serotype, the genotype of ATCC32609 (VGII) is different from WM179 (VGI). That could explain why the cytokine level of IL-12p40 stimulated by ATCC32609 was much lower than WM179. We assume that different genotypes may alter immunological property of Cryptococcus strains. In addition to serotypes, whether Dectin-3 can recognize different genotypes of Cryptococcus still needs further study.

Cryptococcus strains possess the large polysaccharide capsule to shield its cell wall components and the capsule is primarily composed of GXM, which comprises more than $90 \%$ of the capsular polysaccharide mass (29). In this study, both FACS screening and ELISA binding assay showed the direct binding of Dectin-3-Fc fusion protein with all five serotypes of GXM from Cryptococcus capsule. It was also confirmed that the general repeating unit composed of mannan and GluA determines its binding with Dectin-3. Although Dectin-3 can bind all five serotypes of GXM, only GXM-B and GXM-AD are dependent of Dectin-3 recognition to initiate pro-inflammatory responses. There are some possible explanations of this phenomenon. One is that the different quantity and position of xylose may alter the virulence of GXM and the disposition of the $O$-acetyl substituent may determine the antigenic activity (Figure S2E in Supplementary Material). Another reason is that not only Dectin-3, but also other PRRs can recognize Cryptococcus to initiate cell host responses (36).

In pulmonary cryptococcosis model, Dectin-3 deficiency significantly impaired accumulation and killing activity of AMs in lungs. In the meanwhile, Dectin-3-deficient mice had increased fungal burden in lungs. Adoptive transfer has been well established and applied to study the function of specific group of cells (46). In this study, adoptive transfer of WT AMs into Dectin3-deficient mice rescued the number and percentage of AMs in lungs during C.g-B and C.n-AD infection. In consequence, the pro-inflammatory cytokine production increased and fugal burden was decreased. These data indicate that AM plays critical role in clearance of Cryptococcus infection. Complementary to our results, the depletion of AMs prior to infection results in rapid clinical deterioration and death of mice post Cryptococcus infection (47).

It is well-known that CARD9 is an adaptor molecule that plays critical role in anti-fungal immunity and is triggered through CLRs (28) In the present study, CARD9 deficiency impaired 
clearance of C.g-B, C.g-C, C.n-D, and C.n-AD whereas only C.g-B and C.n-AD are dependent of Dectin-3 recognition, which indicates that should be other CLRs that can recognize C.g-C and C.n-D. Dectin-2 is postulated to induce Th2-type responses and IL-4-dependent mucin production in the lungs following infection with C.n-D (20). The impact of Dectin-2 deficiency may also vary depending on the Cryptococcus serotypes.

As it is reported before, CARD9 is also important for recruitment of neutrophils to protect against invasive fungal infection (48). However, the impact of CARD9 on neutrophils recruitment and function against cryptococcal infection seems to be complicated depending on different serotypes of Cryptococcus infection. Besides, DCs are also critical for protection against pulmonary Cryptococcus infection. A recent study reported that plasmacytoid DCs have direct activity against C. neoformans requiring Dectin-3 expression and reactive oxygen species (49). Hence, whether different types of immune cells require Dectin-3 or CARD9 expression to initiate anti-cryptococcal effect deserves further study.

\section{MATERIALS AND METHODS}

\section{Cryptococcus Strain}

Cryptococcus gattii-B strain ATCC32609 and WM179, C. gattiiC strain NIH312, C. neoformans-A strain H99, C. neoformans-D strain WM629, and C. neoformans-AD strain WM628 were gifted from Shanghai institute of fungal medicine, China. The yeast cells were cultured on Sabouraud dextrose agar (SDA) plates before use. Strains cultured in Yeast extract peptone dextrose (YPD) medium at $30^{\circ} \mathrm{C}$ for $16 \mathrm{~h}$, were presented as thin-capsule form. Strains cultured in RPMI-1640 medium plus $10 \% \mathrm{FBS}$ at $37^{\circ} \mathrm{C}$ with $5 \% \mathrm{CO}_{2}$ for $16 \mathrm{~h}$, were presented as thick-capsule form.

\section{BMDM Preparation}

Primary cultures of BMDMs from C57B/L6 mice were prepared as previously described (50). Briefly, bone marrow cells were harvested from the femurs and tibias of mice. Erythrocytes were removed from cells by using a hypotonic solution. Cells were cultured for 7 days in DMEM medium containing 20\% fetal bovine serum, $50 \mathrm{mM} \beta$-mercaptoethanol, $100 \mu \mathrm{g} / \mathrm{mL}$ penicillinstreptomycin, and 30\% conditioned medium from L929 cells expressing M-CSF. On day 3, another $10 \mathrm{~mL}$ of the same medium was added. On day 7 , non-adherent cells were removed and adherent cells were harvested.

\section{Analysis of Chemokine Genes Expression}

Total RNA was extracted from the infected lungs or BMDMs using Triozl (TaKaRa Bio, Otsu, Japan), and the first-strand cDNA was synthesized using PrimeScript first-strand cDNAsynthesis kit (TaKaRa Bio, Otsu, Japan), according to the manufacturer's instructions. Quantitative real-time PCR was performed in a volume of $20 \mu \mathrm{L}$ using gene-specific primers and SYBR Premix Ex TaqII (TaKaRa Bio, Otsu, Japan) in a ABI 7500 system (Applied Biosystems, USA). The amounts of transcript were normalized to GAPDH. $\Delta \Delta \mathrm{Ct}$ method to calculate fold changes. First, $\mathrm{Ct}$ target gene $-\mathrm{Ct}$ housekeeping gene $=\Delta \mathrm{Ct}$. Second, $\Delta \mathrm{Ct}$ treatment $-\Delta$ Ct control $=\Delta \Delta$ Ct. Third, fold changes $=2^{-\Delta \Delta \mathrm{Ct}}$.

\section{Cytokine Assay}

TNF-a, IL-6, IL-12p40, and IL-1 $\beta$ concentrations in the lung homogenates and culture supernatants were measured by SETReady-GO ELISA kits (eBioscience) according to the manufacturer's protocol.

\section{Immunoblot Analysis}

Harvest and pellet BMDM cells. Resuspend cell pellet in $250 \mu \mathrm{L}$ ice cold lysis buffer (10 mM Hepes pH 7.9 + $10 \mathrm{mM} \mathrm{KCl}+0.1 \mathrm{mM}$ EDTA $+0.4 \%$ Nonidet P40) with protease inhibitors (Roche, 1836145) and incubate on ice for $15 \mathrm{~min}$. Spin the lysate for $1 \mathrm{~min}$ at full speed at $4^{\circ} \mathrm{C}$. Transfer supernatant to a fresh tube to keep the cytoplasmic fraction. Wash the nuclear pellet in $500 \mu \mathrm{L}$ ice cold lysis buffer and spin down for $1 \mathrm{~min}$ at $4^{\circ} \mathrm{C}$. Add $20 \mu \mathrm{L}$ extraction buffer $(20 \mathrm{mM}$ Hepes $\mathrm{pH} 7.9+0.4 \mathrm{M} \mathrm{NaCl}+1 \mathrm{mM}$ EDTA) with inhibitors to the pellet and shake vigorously for $15 \mathrm{~min}$ on vortex in cold room. Spin for $10 \mathrm{~min}$ at full speed at $4^{\circ} \mathrm{C}$. Transfer supernatant to fresh tube and store this nuclear extract at $-20^{\circ} \mathrm{C}$. Denature protein before use. Immunoblotting was performed as described previously (27). Anti-IкB $\alpha$ (9247), -phospho-IкB $\alpha$ (9246), -ERK (4372), -phospho-ERK (4695),

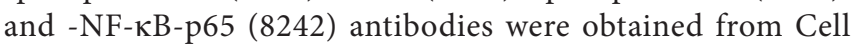
Signaling (Danvers, MA, USA). Anti- $\beta$-actin (ab8224) and -PCNA (ab152112) antibodies were from Abcam (Cambridge, MA, USA).

\section{Isolation and Purification of GXM}

The extracted EPS from Cryptococcus strains were isolated as previously described (51). In detail, strains were grown in YNB culture medium at $30^{\circ} \mathrm{C}$ with shaking for 4 days and then the supernatant were collected after centrifuging with $10,000 \times g$ for $5 \mathrm{~min}$. The supernatant was slowly added with 3 volumes of $\mathrm{EtOH}$ and incubated for overnight at $4^{\circ} \mathrm{C}$, which were centrifuged at $10,000 \times g$ for $10 \mathrm{~min}$. The precipitate was collected for air

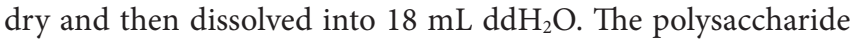
concentration was determined by the phenol sulfuric method of Dubois (data not shown). To purify GXM, the polysaccharide solution was adjusted to $0.2 \mathrm{M} \mathrm{NaCl}$ and finalized with 3 times $(\mathrm{w} / \mathrm{w})$ of $0.3 \%(\mathrm{w} / \mathrm{v}) \mathrm{CTAB}$ solution at room temperature, the mixture of which is named as CTAB-GXM. Then, CTAB-GXM was centrifuged at $10,000 \times g$ for $10 \mathrm{~min}$ and the precipitate of CTAB-GXM was washed with $10 \% \mathrm{EtOH}$ in ddH20 (v/v). To remove CTAB, the precipitate of CTAB-GXM was dissolved in $1 \mathrm{M} \mathrm{NaCl}$ and finalized with about 2.5 times (v/v) of EtOH with stirring. The precipitate containing GXM was collected after centrifuging at $10,000 \times g$ for $10 \mathrm{~min}$ at room temperature and then dissolved in $2 \mathrm{M} \mathrm{NaCl}$ until a viscous solution form. The viscous solution was dialyzed in a dialysis cassette with a 10,000MW cutoff for overnight against $1 \mathrm{M} \mathrm{NaCl}$ and then against fresh $\mathrm{ddH}_{2} \mathrm{O}$ for 1 week. Finally, the solution EPS containing GXM inside the dialysis bag was lyophilized at $-55^{\circ} \mathrm{C}$ for overnight. Endotoxin levels of GXM were measured by endotoxin assay kit and they were all within the detection range of $0.01-1 \mathrm{EU} / \mathrm{mL}$ (Figure S2D in Supplementary Material) (GenScript, Cat. No. L00350C). 


\section{Analysis of Monosaccharide Composition of GXM}

Extracellular polysaccharides $(1 \mathrm{mg})$ was hydrolyzed with $2 \mathrm{M}$ TFA at $120^{\circ} \mathrm{C}$ for $2 \mathrm{~h}$. After repeated evaporation with methanol to completely remove TFA, the residue was dissolved in distilled water and reduced with $\mathrm{NaBH}_{4}$ at room temperature for $3 \mathrm{~h}$. After neutralization with $\mathrm{AcOH}$ and evaporation to dryness, the residue was acetylated with $\mathrm{Ac}_{2} \mathrm{O}$ for $1 \mathrm{~h}$ at $100^{\circ} \mathrm{C}$. The resulting alditol acetates were analyzed with gas chromatography-mass spectrometry (GC-MS). The GC-MS temperature program for monosaccharide analysis was as follows: $140-198^{\circ} \mathrm{C}$ at $2^{\circ} \mathrm{C} / \mathrm{min}$, maintained for $4 \mathrm{~min}$ and increased to $214^{\circ} \mathrm{C}$ at $4^{\circ} \mathrm{C} / \mathrm{min}$, followed by increases of $1^{\circ} \mathrm{C} / \mathrm{min}$ until $217^{\circ} \mathrm{C}$ was reached, which was maintained for $4 \mathrm{~min}$ and finally increased to $250^{\circ} \mathrm{C}$ at $3^{\circ} \mathrm{C} / \mathrm{min}$, maintained for $5 \mathrm{~min}$ as reported previously (52).

\section{Chemical Modification of GXM}

Extracellular polysaccharides were performed with 1-cyclohexyl3-(2-morpholinoethyl) carbodiimide metho- $p$-toluenesulfonate (CMC) and NaBH4 for carboxyl reduction (53). This procedure resulted in D- GluA converted into D-glucose. For deacylation, EPS $(25 \mathrm{mg})$ was treated with $5 \mathrm{~mL} \mathrm{NaOH}(0.1 \mathrm{M})$ for $16 \mathrm{~h}$ at $37^{\circ} \mathrm{C}$, neutralized with $0.1 \mathrm{M} \mathrm{HCl}$, and then dialyzed against water (54).

\section{Nuclear Magnetic Resonance (NMR) Analysis}

Extracellular polysaccharides $(80 \mathrm{mg})$ were dissolved in $0.4 \mathrm{~mL}$ $\mathrm{D}_{2} \mathrm{O}$ (99.8 Atom\% Deuterium; Schweres Wasser, USA). The ${ }^{1} \mathrm{H}$ NMR spectra were measured using the Bruker Avance III 600 Spectrometer (Bruker Instruments, Inc., Billerica, MA, USA) at $25^{\circ} \mathrm{C}$. The chemical shifts of ${ }^{1} \mathrm{H}$ NMR are expressed in ppm by using acetone as an internal standard; $4.70 \mathrm{ppm}$ for ${ }^{1} \mathrm{H}$ NMR. All the experiments were recorded, and data were processed using standard Bruker software and MestReNova (55).

\section{Isolation and Purification of CPS}

The CPS from Cryptococcus strains were isolated as previously described (56). Briefly, Cryptococcus was recovered from $40 \%$ glycerin in $-80^{\circ} \mathrm{C}$ and cultured in YPD (1\% yeast extract, $2 \%$ peptone, $2 \% \mathrm{D}$-glucose, and $0.02 \%$ chloramphenicol) for $18 \mathrm{~h}$. Then, yeast cells were washed three times with sterile PBS and resuspended in RPMI-1640 medium containing 10\% FBS to induce thick capsule in cell incubator for 7 days. After that, yeast cells were collected and suspended in $15 \mathrm{ml}$ of DMSO, incubating for $30 \mathrm{~min}$ twice. Cells were removed by centrifugation at $10,000 \times g$ for $10 \mathrm{~min}$, and the supernatant was dialyzed against by flowing $\mathrm{ddH}_{2} \mathrm{O}$ for 1 day. Finally, the CPS solution inside the dialysis bag was lyophilized.

\section{Plate-Coated GXM}

For cell stimulation, GXM was dissolved in $70 \%$ ethanol at concentration of $500 \mu \mathrm{g} / \mathrm{mL}$. Add $100 \mu \mathrm{L}$ per well to 48 -well cell culture plate and allow ethanol to volatilize, so that GXM was coated on plate. For ELISA binding assay, first, GXM was dissolved in PBS to form soluble polysaccharide solution at concentration of $10 \mu \mathrm{g} / \mathrm{mL}$. Then, add $100 \mu \mathrm{L}$ of GXM solution per well to 96-well ELISA plate and incubate overnight at room temperature. Finally, aspirate and wash three times with PBS.

\section{Expression of Fusion Protein}

The extracellular domain of hDectin-3 was amplified from human PBMC cDNA. The primers were as follows: $\mathrm{F}\left(5^{\prime}-3^{\prime}\right)$ : AGTCTTGCACTTGTCACGAATTCGTGTTTGGTGACTCA TCACAA; R (5'-3'): GCATGTGTGAGT TTTGTCAGATCTGT TCAATGTTGTTCCAGGTA. The PCR program was: $95^{\circ} \mathrm{C}$ $2 \mathrm{~min}, 95^{\circ} \mathrm{C} 20 \mathrm{~s}, 58^{\circ} \mathrm{C} 20 \mathrm{~s}, 72^{\circ} \mathrm{C} 20 \mathrm{~s}, 40$ cycles, $72^{\circ} \mathrm{C} 5 \mathrm{~min}$, and $4^{\circ} \mathrm{C}$ hold. Then, PCR production was purified with PCR Clean Up Kit (AXYGEN). Meanwhile, pFUSE-hIgG1-Fc2 (InvivoGen, USA) were digested by EcoRI (Thermo Fisher, USA) and BglII (Thermo Fisher, USA) about $3 \mathrm{~h}$ at $37^{\circ} \mathrm{C}$, the digesting production was purified with PCR Clean Up Kit. The gene that encoded the Dectin-3 extracellular domains was cloned into pFUSE-hIgG1Fc2 expression vector. Herein, we used homologous recombination enzyme (HanBio, China) to ligate the plasmid and PCR production. The ligated plasmid was transformed into DH5 $\alpha$ (Transgen, China). Select 3-5 bacterial colonies to culture in LB including zeocin (invivogen, USA) over night, the plasmid was obtained according to the manufacture of TIANpure MiNi Plasmid Kit (TINAGEN, China). Finally, CRD-hCLRs-pFUSEhIgG1-Fc2 were sequenced by company (Huagene, China).

The hCLRs-pFUSE-hIgG1-Fc vectors were then transfected into 293T using Lipo2000 (Sigma), 1 day later, adding bleomycin (InvivoGen), the best proportion of bleomycin VS. DMEM is 3:1,000. After culturing 3 days, the cell supernatant was collected and the expression of CLR-Fc fusion protein was determined by immunoblot analysis with anti-Fc antibody.

\section{Fluorescence-Activated Cell-Sorting}

Yeast cells underwent lag phase induction and were plated at $1 \times 10^{7}$ cells/tube. Staining was performed in $0.5 \%$ BSA in PBS. Fusion proteins were then applied at $500 \mu \mathrm{L} /$ tube followed by anti-human FITC-Fc secondary antibody (Abcam, Cambridge, MA, USA). Median fluorescence intensity of each tube was measured by BD FACS Array flow cytometer (BD Biosciences, San Jose, CA, USA) and presented as histogram.

\section{ELISA Binding Assay}

Soluble GXM was adsorbed to ELISA plate at the concentration of $10 \mu \mathrm{g} / \mathrm{mL}$. Then, plate was blocked with $5 \%$ nonfat milk at room temperature for $1 \mathrm{~h}$. Meanwhile, fusion proteins were incubated separately with $\mathrm{PBS} / \mathrm{mannan} / \mathrm{xylose} / \mathrm{GluA}$ (final concentration: $1 \%$ solution) for $30 \mathrm{~min}$ at $37^{\circ} \mathrm{C}$. Then, fusion proteins were added with $100 \mu \mathrm{L} /$ well, incubated at $37^{\circ} \mathrm{C}$ for $2 \mathrm{~h}$, and followed by $100 \mu \mathrm{L} /$ well anti-human HRP-Fc secondary antibody (Jackson ImmunoResearch Laboratories, West Grove, $\mathrm{PA}, \mathrm{USA}$ ) at room temperature for $30 \mathrm{~min}$. There were three times of washing between each step. After that, $100 \mu \mathrm{L} /$ well substrates were added at room temperature for $10 \mathrm{~min}$. Finally, $50 \mu \mathrm{L} /$ well $1 \mathrm{M}$ phosphoric acid was added as stop solution. Test results were measured by plate reader at $450 \mathrm{~nm}$. 


\section{Mice}

Wil-type (WT) C57BL/6, Dectin-3-deficient $\left(\mathrm{Clec} 4 \mathrm{~d}^{-/-}\right)$and CARD9-deficient (CARD9 ${ }^{-/-}$) mice were kept under specificpathogen-free conditions at the Institute for Animal Experimentation, Tongji University School of Medicine. 6- to 8-weekold male and female mice were used in this study. No evidence of susceptibility based on sex was observed.

\section{Pulmonary Cryptococcal Infection in Mice}

Mice were anesthetized by inhaling isoflurane (RWD life science, Shenzhen, China) and restrained on a small board. Live yeast cells $\left(1 \times 10^{5}\right.$ or $\left.1 \times 10^{6}\right)$ were inoculated in a $35 \mu \mathrm{L}$ volume into the trachea of each mouse.

\section{Fungal Burden Analysis}

Mice were sacrificed on day 3 after infection, and lungs or brains were dissected carefully, excised, and then homogenized separately in $1 \mathrm{ml}$ of distilled water by teasing with a stainless mesh at room temperature. The homogenates, diluted appropriately with distilled water, were inoculated at $100 \mu \mathrm{L}$ on SDA plates and cultured for 2 days, and the resulting colonies were counted.

\section{Histological Examination}

The lung specimens obtained from mice were fixed in $10 \%$ buffer formalin, dehydrated, and embedded in paraffin. Sections were cut and stained with hematoxylin \& eosin or Gomoris methenamine silver stain, using standard staining procedures, at pathology platform of Servicebio Technology, Wuhan, China.

\section{Flow Cytometry and Cell Sorting}

Anti-mouse antibodies to CD45-FITC, CD11b-PerCP-Cy5.5, CD11c-APC, Ly6G-BV421, Siglec-F-PE, and fixable viability stain 780 were obtained from BD Pharmingen. The lungs from mice were perfused and digested into single-cell suspensions as described previously (57). After RBC lysis buffer treatment, the whole lung cells were washed with PBS and then stained with corresponding fluorescent antibodies. Following incubation, samples were washed and fixed in $2 \%$ ultrapure formaldehyde. The absolute number of each leukocyte subset was then determined by multiplying the absolute number of $\mathrm{CD} 45^{+}$cells by the percentage of cells stained by fluorochromelabeled antibodies for each cell population analyzed using BD FACSArray software ${ }^{\mathrm{TM}}$ on a BD FACSArray flow cytometer (BD Biosciences, San Jose, CA, USA). AMs were identified as $\mathrm{CD}_{4} 5^{+} \mathrm{CD} 11 \mathrm{c}^{+}$Siglec- $\mathrm{F}^{+}$. Neutrophils were identified as $\mathrm{CD} 45^{+} \mathrm{CD} 11 \mathrm{~b}^{+} \mathrm{Ly}_{6 \mathrm{G}}{ }^{+}$.

Cell sorting was performed on BD FACSAria II instrument, using BD FACSDiva software (BD Biosciences), and compensation and data analyses were performed using FlowJo software (TreeStar, Ashland, OR, USA). Cell populations were identified using sequential gating strategy. The sorting purity was $90-95 \%$.

\section{In Vitro Killing Assay}

Alveolar macrophages were sorted from the lung leukocytes of mice. The purified AMs $\left(1 \times 10^{4} /\right.$ well $)$ were cultured, in triplicate, within individual wells of a 96-well U-bottom tissue culture plate in DMEM complete media containing C.n-A, C.g-B, and C.n-AD respectively at multiplicity of infection of $1,0.1$, and 0.01 ; and with C.n-A, C.g-B, and C.n-AD in DMEM complete media without AMs as a control. After $6 \mathrm{~h}$, the content of each well was centrifuged and the supernatants were removed. The AMs in the cell pellet were then lysed by washing three times with sterile water and incubating in water for $20 \mathrm{~min}$. The remaining yeast was diluted in PBS and plated onto SDA plate to quantify the live cryptococcal cells.

\section{Adoptive Transfer Experiments}

For adoptive transfer experiments, AM $\left(\mathrm{CD} 45^{+} \mathrm{CD} 11 \mathrm{c}^{+}\right.$siglec- $\left.\mathrm{F}^{+}\right)$ from WT and Dectin-3-deficient mice were sorted using a FACSAria II (BD Biosciences). Then freshly sorted AM $\left(5 \times 10^{5}\right.$ cells in $200 \mu \mathrm{L}$ PBS) were transferred intravenously into WT and Dectin-3-deficient mice as described previously (58). At $2 \mathrm{~h}$ after adoptive transfer, mice were challenged with $1 \times 10^{5} \mathrm{CFU}$ of C.g-B strain ATCC32609. At day 1 after challenge, mice were sacrificed and samples were collected for the following experiments.

\section{Statistical Analysis}

At least two biological replicates were performed for all experiments unless otherwise indicated. Log-rank testing was used to evaluate the equality of survival curves. Student's $t$-test for paired observations was used for statistical analyses of cytokine expression levels. Statistical significance was set at a $P$ value of less than $0.05,0.01$, or 0.001 .

\section{ETHICS STATEMENT}

All animal experimental procedures were performed in accordance with the Regulations for the Administration of Affairs Concerning Experimental Animals approved by the State Council of People's Republic of China. The protocol was approved by the Institutional Animal Care and Use Committee of Tongji University (Permit Number: TJLAC-015-002). Human peripheral blood mononuclear cells (PBMCs) were obtained from Department of Medical Laboratory, Shanghai Pulmonary Hospital of China.

\section{AUTHOR CONTRIBUTIONS}

H-RH, J-FX, and X-MJ designed the experiments. H-RH, FL, $\mathrm{HH}, \mathrm{XX}, \mathrm{NL}$, and SW performed the experiments. H-RH, J-FX, and $\mathrm{X}-\mathrm{MJ}$ analyzed the data and wrote the manuscript with editorial input from all the authors.

\section{ACKNOWLEDGMENTS}

We thank Dr. Xin Lin and Dr. Xue-Qiang Zhao for helpful advice and suggestions. This work was supported by the National Natural Science Foundation of China (31622023 and 81571611 to X-MJ, 81670006 to J-FX), Shanghai leading talent Program (2016036 to J-FX), Shanghai laboratory animal research fund 
(16140902600 to X-MJ), Outstanding academic leaders of Shanghai health and Family Planning Commission (2017BR024 to X-MJ), and Shuguang Program of Shanghai Education Development Foundation and Shanghai Municipal Education Commission (17SG24 to X-MJ).

\section{REFERENCES}

1. Idnurm A, Bahn YS, Nielsen K, Lin X, Fraser JA, Heitman J. Deciphering the model pathogenic fungus Cryptococcus neoformans. Nat Rev Microbiol (2005) 3:753. doi:10.1038/nrmicro1245

2. Kwon-Chung KJ, Boekhout T, Fell JW, Diaz M. (1557) Proposal to conserve the name Cryptococcus gattii against C. hondurianus and C. bacillisporus (Basidiomycota, Hymenomycetes, Tremellomycetidae). Taxon (2002) 51: 804-6. doi:10.2307/1555045

3. Dromer F, Gueho E, Ronin O, Dupont B. Serotyping of Cryptococcus neoformans by using a monoclonal antibody specific for capsular polysaccharide. J Clin Microbiol (1993) 31:359-63.

4. Ikeda R, Nishimura S, Nishikawa A, Shinoda T. Production of agglutinating monoclonal antibody against antigen 8 specific for Cryptococcus neoformans serotype D. Clin Diagn Lab Immunol (1996) 3:89-92.

5. Mitchell TG, Perfect JR. Cryptococcosis in the era of AIDS - 100 years after the discovery of Cryptococcus neoformans. Clin Microbiol Rev (1995) 8:515-48.

6. Dromer F, Mathoulin-Pélissier S, Launay O, Lortholary O, Group FCS. Determinants of disease presentation and outcome during cryptococcosis: the CryptoA/D study. PLoS Med (2007) 4:e21. doi:10.1371/journal.pmed. 0040021

7. Viviani MA, Cogliati M, Esposto MC, Lemmer K, Tintelnot K, Colom Valiente MF, et al. Molecular analysis of 311 Cryptococcus neoformans isolates from a 30-month ECMM survey of cryptococcosis in Europe. FEMS Yeast Res (2006) 6:614-9. doi:10.1111/j.1567-1364.2006.00081.x

8. Galanis E, Hoang L, Kibsey P, Morshed M, Phillips P. Clinical presentation, diagnosis and management of Cryptococcus gattii cases: lessons learned from British Columbia. Can J Infect Dis Med Microbiol (2009) 20:23-8.

9. Kwon-Chung K, Bennett J. Epidemiologic differences between the two varieties of Cryptococcus neoformans. Am J Epidemiol (1984) 120:123-30. doi:10.1093/oxfordjournals.aje.a113861

10. Kidd S, Hagen F, Tscharke R, Huynh M, Bartlett K, Fyfe M, et al. A rare genotype of Cryptococcus gattii caused the cryptococcosis outbreak on Vancouver Island (British Columbia, Canada). Proc Natl Acad Sci U S A (2004) 101:17258-63. doi:10.1073/pnas.0402981101

11. Byrnes EJ III, Li W, Lewit Y, Ma H, Voelz K, Ren P, et al. Emergence and pathogenicity of highly virulent Cryptococcus gattii genotypes in the northwest United States. PLoS Pathog (2010) 6:e1000850. doi:10.1371/ journal.ppat.1000850

12. Goldman DL, Khine H, Abadi J, Lindenberg DJ, Pirofski La, Niang R, et al. Serologic evidence for Cryptococcus neoformans infection in early childhood. Pediatrics (2001) 107:E66. doi:10.1542/peds.107.5.e66

13. Hardison SE, Brown GD. C-type lectin receptors orchestrate antifungal immunity. Nat Immunol (2012) 13:817-22. doi:10.1038/ni.2369

14. Geijtenbeek TB, Gringhuis SI. C-type lectin receptors in the control of T helper cell differentiation. Nat Rev Immunol (2016) 16:433-48. doi:10.1038/ nri.2016.55

15. Taylor PR, Tsoni SV, Willment JA, Dennehy KM, Rosas M, Findon H, et al. Dectin-1 is required for beta-glucan recognition and control of fungal infection. Nat Immunol (2007) 8:31-8. doi:10.1038/ni1408

16. Steele C, Rapaka RR, Metz A, Pop SM, Williams DL, Gordon S, et al. The beta-glucan receptor dectin-1 recognizes specific morphologies of Aspergillus fumigatus. PLoS Pathog (2005) 1:e42. doi:10.1371/journal.ppat. 0010042

17. Nakamura K, Kinjo T, Saijo S, Miyazato A, Adachi Y, Ohno N, et al. Dectin-1 is not required for the host defense to Cryptococcus neoformans. Microbiol Immunol (2007) 51:1115-9. doi:10.1111/j.1348-0421.2007.tb04007.x

18. Saijo S, Ikeda S, Yamabe K, Kakuta S, Ishigame H, Akitsu A, et al. Dectin-2 recognition of alpha-mannans and induction of Th17 cell differentiation

\section{SUPPLEMENTARY MATERIAL}

The Supplementary Material for this article can be found online at https://www.frontiersin.org/articles/10.3389/fimmu.2018.01781/ full\#supplementary-material.

is essential for host defense against Candida albicans. Immunity (2010) 32:681-91. doi:10.1016/j.immuni.2010.05.001

19. Loures FV, Röhm M, Lee CK, Santos E, Wang JP, Specht CA, et al. Recognition of Aspergillus fumigatus hyphae by human plasmacytoid dendritic cells is mediated by dectin-2 and results in formation of extracellular traps. PLoS Pathog (2015) 11:e1004643. doi:10.1371/journal.ppat.1004643

20. Nakamura Y, Sato K, Yamamoto H, Matsumura K, Matsumoto I, Nomura T, et al. Dectin-2 deficiency promotes $T h 2$ response and mucin production in the lungs after pulmonary infection with Cryptococcus neoformans. Infect Immun (2015) 83:671-81. doi:10.1128/IAI.02835-14

21. Ishikawa T, Itoh F, Yoshida S, Saijo S, Matsuzawa T, Gonoi T, et al. Identification of distinct ligands for the C-type lectin receptors Mincle and Dectin-2 in the pathogenic fungus Malassezia. Cell Host Microbe (2013) 13: 477-88. doi:10.1016/j.chom.2013.03.008

22. Yonekawa A, Saijo S, Hoshino Y, Miyake Y, Ishikawa E, Suzukawa M, et al. Dectin-2 is a direct receptor for mannose-capped lipoarabinomannan of mycobacteria. Immunity (2014) 41:402-13. doi:10.1016/j.immuni.2014. 08.005

23. Zhu LL, Zhao XQ, Jiang C, You Y, Chen XP, Jiang YY, et al. C-type lectin receptors Dectin-3 and Dectin-2 form a heterodimeric pattern-recognition receptor for host defense against fungal infection. Immunity (2013) 39: 324-34. doi:10.1016/j.immuni.2013.05.017

24. Zhao XQ, Zhu LL, Chang Q, Jiang C, You Y, Luo T, et al. C-type lectin receptor dectin-3 mediates trehalose 6,6'-dimycolate (TDM)-induced Mincle expression through CARD9/Bcl10/MALT1-dependent nuclear factor (NF)-kappaB activation. J Biol Chem (2014) 289:30052-62. doi:10.1074/jbc. M114.588574

25. Campuzano A, Castro-Lopez N, Wozniak KL, Leopold Wager CM, Wormley FL Jr. Dectin-3 is not required for protection against Cryptococcus neoformans infection. PLoS One (2017) 12:e0169347. doi:10.1371/journal. pone. 0169347

26. Gross O, Gewies A, Finger K, Schäfer M, Sparwasser T, Peschel C, et al. Card 9 controls a non-TLR signalling pathway for innate anti-fungal immunity. Nature (2006) 442:651-6. doi:10.1038/nature04926

27. Jia XM, Tang B, Zhu LL, Liu YH, Zhao XQ, Gorjestani S, et al. CARD9 mediates Dectin-1-induced ERK activation by linking Ras-GRF1 to H-Ras for antifungal immunity. J Exp Med (2014) 211:2307-21. doi:10.1084/jem. 20132349

28. Yamamoto H, Nakamura Y, Sato K, Takahashi Y, Nomura T, Miyasaka T, et al. Defect of CARD9 leads to impaired accumulation of gamma interferonproducing memory phenotype $\mathrm{T}$ cells in lungs and increased susceptibility to pulmonary infection with Cryptococcus neoformans. Infect Immun (2014) 82:1606-15. doi:10.1128/IAI.01089-13

29. Zaragoza O, Rodrigues ML, De Jesus M, Frases S, Dadachova E, Casadevall A. The capsule of the fungal pathogen Cryptococcus neoformans. Adv Appl Microbiol (2009) 68:133-216. doi:10.1016/S0065-2164(09)01204-0

30. Doering TL. How does Cryptococcus get its coat? Trends Microbiol (2000) 8:547-53. doi:10.1016/S0966-842X(00)01890-4

31. Bhattacharjee AK, Bennett JE, Glaudemans CP. Capsular polysaccharides of Cryptococcus neoformans. Rev Infect Dis (1984) 6:619-24. doi:10.1093/ clinids/6.5.619

32. Cherniak R, Valafar H, Morris LC, Valafar F. Cryptococcus neoformans chemotyping by quantitative analysis of $1 \mathrm{H}$ nuclear magnetic resonance spectra of glucuronoxylomannans with a computer-simulated artificial neural network. Clin Diagn Lab Immunol (1998) 5:146-59.

33. Belay T, Cherniak R. Determination of antigen binding specificities of Cryptococcus neoformans factor sera by enzyme-linked immunosorbent assay. Infect Immun (1995) 63:1810-9.

34. Shoham S, Huang C, Chen JM, Golenbock DT, Levitz SM. Toll-like receptor 4 mediates intracellular signaling without TNF-alpha release in response 
to Cryptococcus neoformans polysaccharide capsule. J Immunol (2001) 166:4620-6. doi:10.4049/jimmunol.166.7.4620

35. Yauch LE, Mansour MK, Shoham S, Rottman JB, Levitz SM. Involvement of CD14, toll-like receptors 2 and 4, and MyD88 in the host response to the fungal pathogen Cryptococcus neoformans in vivo. Infect Immun (2004) 72:5373-82. doi:10.1128/IAI.72.9.5373-5382.2004

36. Fonseca FL, Nohara LL, Cordero RJ, Frases S, Casadevall A, Almeida IC, et al. Immunomodulatory effects of serotype B glucuronoxylomannan from Cryptococcus gattii correlate with polysaccharide diameter. Infect Immun (2010) 78:3861-70. doi:10.1128/IAI.00111-10

37. Nakamura K, Miyagi K, Koguchi Y, Kinjo Y, Uezu K, Kinjo T, et al. Limited contribution of toll-like receptor 2 and 4 to the host response to a fungal infectious pathogen, Cryptococcus neoformans. FEMS Immunol Med Microbiol (2006) 47:148-54. doi:10.1111/j.1574-695X.2006.00078.x

38. Biondo C, Midiri A, Messina L, Tomasello F, Garufi G, Catania MR, et al. MyD88 and TLR2, but not TLR4, are required for host defense against Cryptococcus neoformans. Eur JImmunol (2005) 35:870-8. doi:10.1002/eji. 200425799

39. Love GL, Boyd GD, Greer DL. Large Cryptococcus neoformans isolated from brain abscess. J Clin Microbiol (1985) 22:1068-70.

40. Bacon BE, Cherniak R, Kwon-Chung KJ, Jacobson ES. Structure of the O-deacetylated glucuronoxylomannan from Cryptococcus neoformans Cap70 as determined by 2D NMR spectroscopy. Carbohydr Res (1996) 283:95-110. doi:10.1016/0008-6215(95)00397-5

41. Brummer E. Human defenses against Cryptococcus neoformans: an update. Mycopathologia (1998) 143:121-5. doi:10.1023/A:1006905331276

42. Wang H, Li M, Lerksuthirat T, Klein B, Wuthrich M. The C-type lectin receptor MCL mediates vaccine-induced immunity against infection with Blastomyces dermatitidis. Infect Immun (2015) 84:635-42. doi:10.1128/IAI. 01263-15

43. Metz H. [Contribution to the morphology \& biology of the lithium \& cesium variants of Salmonella paratyphi B]. Zentralbl Bakteriol Orig (1958) 171:461-9.

44. Kwon-Chung KJ, Fraser JA, Doering TL, Wang Z, Janbon G, Idnurm A, et al. Cryptococcus neoformans and Cryptococcus gattii, the etiologic agents of cryptococcosis. Cold Spring Harb Perspect Med (2014) 4:a019760. doi:10.1101/ cshperspect.a019760

45. Perfect JR, Bicanic T. Cryptococcosis diagnosis and treatment: what do we know now. Fungal Genet Biol (2015) 78:49-54. doi:10.1016/j.fgb.2014.10.003

46. Xu X, Xu JF, Zheng G, Lu HW, Duan JL, Rui W, et al. CARD9(S12N) facilitates the production of IL- 5 by alveolar macrophages for the induction of type 2 immune responses. Nat Immunol (2018) 19:547-60. doi:10.1038/ s41590-018-0112-4

47. Osterholzer JJ, Milam JE, Chen GH, Toews GB, Huffnagle GB, Olszewski MA. Role of dendritic cells and alveolar macrophages in regulating early host defense against pulmonary infection with Cryptococcus neoformans. Infect Immun (2009) 77:3749-58. doi:10.1128/IAI.00454-09

48. Drummond RA, Collar AL, Swamydas M, Rodriguez CA, Lim JK, Mendez LM, et al. CARD9-dependent neutrophil recruitment protects against fungal invasion of the central nervous system. PLoS Pathog (2015) 11:e1005293. doi:10.1371/journal.ppat.1005293

49. Hole CR, Leopold Wager CM, Mendiola AS, Wozniak KL, Campuzano A, Lin $\mathrm{X}$, et al. Antifungal activity of plasmacytoid dendritic cells against Cryptococcus neoformans in vitro requires expression of Dectin-3 (CLEC4D) and reactive oxygen species. Infect Immun (2016) 84:2493-504. doi:10.1128/ IAI.00103-16

50. Bi L, Gojestani S, Wu W, Hsu YM, Zhu J, Ariizumi K, et al. CARD9 mediates dectin-2-induced IkappaBalpha kinase ubiquitination leading to activation of NF-kappaB in response to stimulation by the hyphal form of Candida albicans. J Biol Chem (2010) 285:25969-77. doi:10.1074/jbc.M110.131300

51. Wozniak KL, Levitz SM. Isolation and purification of antigenic components of Cryptococcus. Methods Mol Biol (2009) 470:71-83. doi:10.1007/9781-59745-204-5_7

52. Chen M, Wu J, Shi S, Chen Y, Wang H, Fan H, et al. Structure analysis of a heteropolysaccharide from Taraxacum mongolicum Hand.-Mazz. and anticomplementary activity of its sulfated derivatives. Carbohydr Polym (2016) 152:241-52. doi:10.1016/j.carbpol.2016.06.110

53. Richards JC, Perry MB, Kniskern PJ. Structural analysis of the specific polysaccharide of Streptococcus pneumoniae type 9L (American type 49). Can J Biochem Cell Biol (1984) 62:1309-20. doi:10.1139/o84-167

54. Urai M, Anzai H, Ogihara J, Iwabuchi N, Harayama S, Sunairi M, et al. Structural analysis of an extracellular polysaccharide produced by Rhodococcus rhodochrous strain S-2. Carbohydr Res (2006) 341:766-75. doi:10.1016/j. carres.2006.01.010

55. Wang H, Shi S, Bao B, Li X, Wang S. Structure characterization of an arabinogalactan from green tea and its anti-diabetic effect. Carbohydr Polym (2015) 124:98-108. doi:10.1016/j.carbpol.2015.01.070

56. Bryan RA, Zaragoza O, Zhang T, Ortiz G, Casadevall A, Dadachova E. Radiological studies reveal radial differences in the architecture of the polysaccharide capsule of Cryptococcus neoformans. Eukaryot Cell (2005) 4:465-75. doi:10.1128/EC.4.2.465-475.2005

57. Sauer KA, Scholtes P, Karwot R, Finotto S. Isolation of CD4+ T cells from murine lungs: a method to analyze ongoing immune responses in the lung. Nat Protoc (2006) 1:2870-5. doi:10.1038/nprot.2006.435

58. Zhao X, Guo Y, Jiang C, Chang Q, Zhang S, Luo T, et al. JNK1 negatively controls antifungal innate immunity by suppressing CD23 expression. Nat Med (2017) 23:337-46. doi:10.1038/nm.4260

Conflict of Interest Statement: The authors declare that the research was conducted in the absence of any commercial or financial relationships that could be construed as a potential conflict of interest.

Copyright $\odot 2018$ Huang, Li, Han, Xu, Li, Wang, Xu and Jia. This is an open-access article distributed under the terms of the Creative Commons Attribution License (CC BY). The use, distribution or reproduction in other forums is permitted, provided the original author(s) and the copyright owner(s) are credited and that the original publication in this journal is cited, in accordance with accepted academic practice. No use, distribution or reproduction is permitted which does not comply with these terms. 